\title{
Effect of Evaporating Sea Spray on Heat Fluxes in a Marine Atmospheric Boundary Layer
}

\author{
YEVGENII RASTIGEJEV \\ North Carolina A\&T State University, Greensboro, North Carolina \\ SERGEY A. SUSLOV \\ Swinburne University of Technology, Hawthorn, Victoria, Australia
}

(Manuscript received 13 November 2018, in final form 8 May 2019)

\begin{abstract}
A detailed analysis of the evaporating ocean spray effect on the vertical latent and sensible heat fluxes in a marine atmospheric boundary layer (MABL) for different droplet sizes, vertical distributions of air temperature, humidity, and turbulent intensity is presented. For our analysis we have employed a twotemperature nonequilibrium MABL model developed in our previous work. The obtained analytical and numerical solutions show that the latent and total heat fluxes are significantly enhanced by large droplets because these droplets produce steep vertical gradients of moisture and air temperature in a MABL. Small droplets, however, do not noticeably change the total heat flux but rather redistribute the energy between its sensible and latent components. It has been shown that evaporating spray affects the turbulent kinetic energy (thus the intensity of the vertical turbulent transport) mostly mechanically by altering the vertical distribution of the mass density of the air-spray mixture rather than thermodynamically by changing vertical profiles of the air temperature and moisture. Furthermore, we have found that the vertical profiles of heat fluxes are approximately self-similar for a wide range of defining parameters, that is, can be approximately scaled to a reference heat profile for a wide range of vertical distributions of the temperature, humidity, and turbulence intensity. The obtained analytical expressions for the vertical heat fluxes affected by the spray presence enable their quick and efficient calculations. This will allow for the future construction of a computationally efficient spray and accurate parameterization to be used in global weather prediction models.
\end{abstract}

\section{Introduction}

Forecasting tropical cyclones (TCs) includes two main tasks-predicting their track and intensity. Even though TC track forecasting has been noticeably improving over past decades, the progress in forecasting of cyclone intensity remains limited, particularly due to the lack of understanding of the air-sea turbulent exchange of sensible and latent heat and momentum in a spray-laden marine atmospheric boundary layer (MABL). The TC intensity depends on these fluxes because a tropical storm gains its energy primarily via a flux of heat derived from the ocean and loses it via momentum transport to the ocean. This idea has been used by Emanuel and his coauthors (Emanuel 1986, 1995, 1997; Bister and Emanuel 1998) to conclude that higher heat exchange and lower drag are essential to intensify and sustain a hurricane.

Corresponding author: Yevgenii Rastigejev, yarastig@ncat.edu
There is a significant evidence based on measurements and theoretical modeling that ocean spray plays an important role in the air-sea interaction affecting a hurricane both mechanically and thermodynamically (Lighthill 1999; Bao et al. 2000; Andreas and Decosmo 2002; Drennan et al. 2007; Gall et al. 2008; Zhang and Perrie 2008; Bianco et al. 2011; Jeong et al. 2012; Shpund et al. 2012; Rastigejev et al. 2011; Rastigejev and Suslov 2014; Wu et al. 2015; Chen and Yu 2016; Zhang et al. 2016; Rastigejev and Suslov 2016; Tang et al. 2017; Peng and Richter 2017). However, the influence of spray on hurricane dynamics is still not fully understood or properly quantified largely due to the difficulties associated with a mathematical description of a spray-laden turbulent MABL in high-wind conditions. Furthermore, it is very difficult to carry out reliable measurements in these conditions (Black et al. 2007; Powell et al. 2003; Jarosz et al. 2007).

In this paper we focus on the effect of evaporating ocean spray on heat fluxes in a MABL. Even though it has been intensively studied (Ling and Kao 1976; Bortkovskii 
1987; Rouault et al. 1991; Edson et al. 1996; Makin 1998; Toffoli et al. 2011; Bao et al. 2011; Bianco et al. 2011; Shpund et al. 2011, 2012, 2014; Mueller and Veron 2014; Rastigejev and Suslov 2016; He et al. 2018) over the past five decades, its understanding and quantification are still limited. As a result of that, numerical experiments with spray parameterizations integrated into bigger numerical weather prediction (NWP) codes (Fairall et al. 1994; Andreas and Decosmo 1999; Bao et al. 2000; Perrie et al. 2005; Gall et al. 2008) have produced several interesting but sometimes contradictory and disputable outcomes. For example, computational experiments with a highresolution TC model (Wang et al. 2001) have demonstrated that parameterizations suggested by Fairall et al. (1994) and Andreas and Decosmo (1999) yield significantly different results for the vertical fluxes of heat and momentum. Bao et al. (2000) reported that sea spray evaporation can increase the intensity of a TC only when a small fraction of the total spray mass evaporates, but when the evaporating fraction of sea spray increases, its impact on hurricane intensity is reduced. Contrary to this result, Gall et al. (2008) concluded that sea spray can affect the hurricane structure and dynamics strongly only if its concentration is high. Therefore, even though there is a general consensus in the literature that spray has a strong effect on heat and momentum transport in hurricanes, a clear understanding of the dynamics of a MABL laden with evaporating spray is still lacking and further systematic studies of this subject are needed.

Recently, the authors have developed a two-temperature nonequilibrium model of a MABL laden with evaporating spray (further referred to as RS16) to describe the spray effect on hurricane dynamics (Rastigejev and Suslov 2016). Mathematically, the model is formulated as a boundary value problem for a system of conservation equations of mass, momentum, and thermal and turbulent kinetic energies for both gas and liquid phases. The model uses an Eulerian-Eulerian multi-fluid-type formulation that considers both liquid and gas phases as continuous interpenetrating media. It consistently describes a two-way coupling between mechanical and thermodynamic influences of the ocean spray and accounts for various other factors (e.g., the size of the evaporating droplets, their concentration, vertical variation of temperature and moisture). Recently, computational models taking into account various physical factors have been also developed by other authors, for example, Pinsky et al. (2008), Magaritz et al. (2009), and Shpund et al. (2011, 2012, 2014). Specifically, these authors focused on the influence on a MABL exerted by the presence of large convective structures that can create an updraft strong enough to carry a significant amount of sea spray to heights corresponding to the lower edge of clouds forming in a hurricane. While including many microphysics effects such a model has an obvious shortcoming: apparently its mathematical formulation is too complicated so that only a qualitative verbal description of its elements could be given in the referenced articles, which naturally limits the use and verification of this model by other researchers.

In developing our model we chose a different approach. Mathematically, our model is represented by a system of explicitly given ordinary differential equations (ODE) describing the vertical distribution of horizontally averaged quantities characterizing a hurricane treating the atmospheric boundary layer as a two-phase system. This enables us to consider separate heat flux components associated with moist air and liquid spray and thus clearly distinguish between them without numerical complications typically associated with a discrete (spectral) treatment of spray droplets. While the derived equations may still look somewhat involved, they are presented in full and they only require on the order of a minute or less time to be solved numerically with a single CPU unit using standard routines available in many widely used software packages such as MATLAB. The ODE formulation also enables us to develop analytic self-similar and asymptotic solutions in important limiting cases such as the small spray concentration limit. Numerical evaluation of such solutions takes just a fraction of a second on a desktop computer making them very attractive for the use in MABL parameterization in global weather prediction codes, the feature that could not be feasible for computationally heavy multidimensional numerical simulations of a MABL such as reported, for example, in Shpund et al. (2011, 2012, 2014).

Several other approaches to modeling spray influence on the marine boundary layer have been suggested in literature to date. They generally fall into two categories of Lagrangian and Eulerian descriptions. A good concise summary of these can be found, for example, in Shpund et al. (2011). Due to the complexity and the large number of interacting physical processes taking place in a MABL, invariably, all existing models include various empirical correlations and simplifying assumptions. Both assumptions and correlations vary from model to model and the current lack of comprehensive field observation data for tropical storms and hurricanes makes it difficult to objectively validate the used simplifications. This in turn makes an attempt of judging the superiority of one model over the other a rather hypothetical and subjective exercise. To make the situation worse, the used assumptions are frequently hard-wired in a particular heavy computational code and thus cannot be replaced or removed without destroying the complete model should it become known that they are not sufficiently accurate to represent a physical reality. 
Being aware of these difficulties, when developing our model we consistently adhered to two main principles. The first is that the major model blocks that we develop are derived from the fundamental principles of conservation of mass, momentum, and energy written in their exact correlation-free mathematical form. We avoid "black boxes" and ad hoc correlations and aim to report sufficient amount of algebraic details for an interested reader to be able to follow through and verify the derivations. Whenever the current state of knowledge does not allow us to introduce a rigorous mathematical description of a particular physical feature or process, we ensure that any empirical formulations are introduced in our model in a modular or parametric form that can be easily replaced if and when the more accurate description of such features or processes becomes available without the need of redeveloping the complete model. In particular, currently we use the standard $E-\varepsilon$ turbulence closure because at the time of writing this was one of the most well-tested turbulence models available for multiphase flow modeling (Jakobsen 2008) that is also widely used for atmospheric boundary layer simulation (Stull 1988). However, it would be very straightforward to replace it with a more accurate or suitable closure should it become available in the future. The other example of a robust structure of the model suggested here is the parametric introduction of the spray concentration $s_{0}$ at the wave-crest level. Its exact value and its dependence on the wind speed in realistic hurricane conditions is currently unknown even though several correlation functions have been suggested, see the review of available data in Rastigejev and Suslov (2014) and the description of assumed correlations in Shpund et al. (2014) and Fairall et al. (2009). The current treatment of $s_{0}$ as a free parameter will enable one to incorporate any reliable dependency of the spray production rate on the particular hurricane conditions determined in the future field observations or obtained theoretically/numerically without breaking the integrity of the present model.

The modular structure of the current model has a further advantage. The modules describing various physical processes can be added incrementally. This enables one to distinguish the influences of individual physical factors. Using this feature in the current work, we have extended the RS16 model to account for the sensible heat flux in the reference spray-free atmosphere and carried out an extensive numerical study of the effect of evaporating ocean spray on the vertical latent and sensible heat fluxes in a spray-laden MABL. Namely, in our previous paper we analyzed only the atmosphere laden with evaporating spray where the vertical sensible heat flux was caused solely by the presence of spray. In the current work we have extended our consideration to the atmosphere, where the sensible heat flux is a sum of the two components- the nonzero sensible heat flux in the corresponding reference spray-free atmosphere and the spray-induced sensible heat flux. Furthermore, we have derived a reduced mathematical model, which is valid for low-to-moderate spray concentrations. The relative simplicity of this model enables us to carry out its asymptotic analysis (Bender and Orszag 1999; Paulsen 2013) and obtain analytic expressions for the vertical distributions of flow characteristics and heat fluxes. The obtained analytical expressions serve several purposes. First, they enable us to validate our numerical code by comparing the analytical and numerical results. Second, a study of the obtained analytical functions in conjunction with numerically generated data provides a much deeper insight into the physics of the problem than numerical data alone. In particular, we have demonstrated that the vertical profiles of heat fluxes are approximately self-similar, that is, they can be scaled to a reference heat flux profile for a wide range of defining parameters. Third, the obtained analytical expressions for heat fluxes affected by the spray presence enable their quick and efficient calculation. This will allow us to construct accurate and computationally efficient spray parameterization schemes employed in large-scale NWP models in the future.

We have found that coupling between the thermodynamic and mechanical effects of spray is not symmetric. For example, suppression of turbulence caused by the mechanical influence of spray does affect heat fluxes. On the other hand, changes in thermodynamic characteristics of a MABL due to spray have only a weak effect on the turbulence intensity and wind velocity. Therefore, the mechanical effect of spray can be studied separately from the thermodynamic one as indeed has been done in our previous reports (see Rastigejev et al. 2011; Rastigejev and Suslov 2014, 2016). In the present work we consider both effects but when analyzing the spray impact on heat fluxes we focus only on the most important physical factors. Specifically, we investigate the influences of the spray concentration and the size of evaporating droplets under various atmospheric (different vertical distributions of temperature, moisture, and turbulence intensity) and ocean surface (surface temperature and pressure) conditions.

We have shown that the specific humidity and the air temperature are more strongly affected by small droplets because of their intensive evaporation and heat exchange with the ambient air throughout the complete domain. At the same time their effect on the total heat flux is not as profound: fine spray primarily redistributes thermal energy between its latent and sensible heat components. The effect of large droplets is felt mostly 
in a thin layer near the ocean surface where most of them reside. Their evaporation, the associated sensible heat exchange and the suppression of turbulent intensity there leads to steep gradients in the vertical distributions of the moisture and the air temperature. As a result, large droplets noticeably enhance the total and latent heat fluxes. This enhancement of the total heat flux by large droplets has also been argued qualitatively by Peng and Richter (2017) even though it was not strong for the cases considered in their paper. In the present work we have carried out a detailed numerical study of the total heat flux for a range of droplet sizes. We have shown that the enhancement of the total heat flux due to spray is significant even for a moderate amount of spray and it reaches its maximum for very large droplets with radius of $\sim 0.5 \mathrm{~mm}$.

We have compared the results of calculation for the vertical heat flux produced by the present spray model with those derived from observations (Bell et al. 2012) for wind speeds greater than $50 \mathrm{~m} \mathrm{~s}^{-1}$. In particular, Bell et al. (2012) showed that the total vertical enthalpy flux grows much faster with the wind speed than predicted by the theory of a turbulent boundary layer. Our spray model calculations agree very well with the data reported by Bell et al. (2012). Therefore, the presence of spray in a MABL may explain the fast growth of the vertical heat flux with the wind speed.

The rest of this paper is organized as follows. Section 2 presents the governing equations. Section 3 describes semi-self-similar vertical profiles of latent and sensible heat fluxes. Section 4 describes the reduced mathematical model. Section 5 discusses numerical and analytical results. Section 6 summarizes the main conclusions of the paper. The definition of boundary parameters, derivation of semi-self-similar distributions of heat fluxes, and asymptotic solutions for the reduced model are presented in appendixes A-C.

\section{Full mathematical model}

\section{a. Governing equations}

Below we briefly describe the two-temperature nonequilibrium model of a MABL laden with evaporating spray introduced in Rastigejev and Suslov (2016). The governing equations of the model describe the variation of the nondimensional mean values of sea spray volume fraction $s$, the specific humidity $q$, the potential $\theta$ and standard $T_{a}$ temperatures of air, the spray water temperature $T_{w}$, the turbulent kinetic energy $e$, its dissipation rate $\varepsilon$, the wind speed $u$, and the atmospheric pressure $P$ with the vertical coordinate $z$ measured from the mean sea surface level, respectively. The equations are written in the nondimensional form as

$$
\begin{aligned}
\frac{d}{d z}\left(k \frac{d s}{d z}+\pi_{1} s\right) & =-\pi_{2} q_{w} s-\pi_{1} \delta_{z-1}, \\
\frac{d}{d z}\left(k \rho_{a} \frac{d q}{d z}\right) & =\pi_{3} q_{w} s, \\
\frac{d}{d z}\left(k \rho_{d} \frac{d \theta}{d z}\right) & =\pi_{5}\left(T_{a}-T_{w}\right) s, \\
\frac{d}{d z}\left(s k \frac{d T_{w}}{d z}+\pi_{1} H_{z-1} T_{w}\right) & =\left[\pi_{6}\left(T_{w}-T_{a}\right)-\pi_{7} q_{w}\right] s \\
& -\pi_{1} \delta_{z-1}, \\
\frac{d}{d z}\left(k \rho \frac{d e}{d z}\right) & =\frac{\rho}{\alpha_{e}}(\varepsilon-p), \\
\frac{d}{d z}\left(k \rho \frac{d \varepsilon}{d z}\right) & =\frac{\rho}{\alpha_{\varepsilon}} \frac{\varepsilon}{e}\left(C_{\varepsilon, 2} \varepsilon-C_{\varepsilon, 1} p\right), \\
\frac{d u}{d z} & =\frac{1}{k \rho}, \quad \text { and } \\
\frac{d P}{d z} & =-\pi_{8} \rho,
\end{aligned}
$$

where

$$
\begin{aligned}
k & =\frac{(\alpha e)^{2}}{\varepsilon}, \quad q_{w}=\frac{S-1}{1-S_{0}} \frac{1}{F_{k}+F_{d}}, \quad S=\frac{P}{p_{v, \mathrm{sat}}} \frac{q}{1+\pi_{9} q}=\frac{q}{q_{\mathrm{sat}}} \frac{1+\pi_{9} q_{\mathrm{sat}}}{1+\pi_{9} q}, \quad F_{k}=\frac{1}{T_{a}^{2}}, \quad F_{d}=\pi_{10} \frac{T_{a}}{p_{v, \mathrm{sat}}}, \\
p_{v, \mathrm{sat}}\left(T_{a}\right) & =T_{a}^{-k_{1}} \exp \left[\frac{k_{2}}{T_{a}}\left(T_{a}-1\right)\right], \quad \theta=\frac{T_{a}}{P^{\kappa_{d}}}, \quad \theta_{v}=\left(1+\pi_{9} q\right) \frac{T_{a}}{P^{\kappa_{d}}}, \quad p=\frac{1}{k \rho^{2}}+\pi_{12} \frac{k}{\rho} \frac{d s}{d z}-\pi_{13} \frac{k}{\theta_{v}} \frac{d \theta_{v}}{d z}, \\
\rho_{a} & =\frac{P}{T_{a}} \frac{1}{1+\pi_{9} q}, \quad \rho_{d}=\rho_{a}\left(1-q_{0} q\right), \quad \rho=\rho_{a}+\pi_{14} s .
\end{aligned}
$$

The governing equations are analyzed subject to the following boundary conditions: 
TABLE 1. Parameters appearing in the model.

\begin{tabular}{|c|c|c|c|}
\hline Parameter & Meaning & Parameter & Meaning \\
\hline$F_{d}$ & $\begin{array}{l}\text { Thermal resistance due } \\
\text { to water vapor diffusion }\end{array}$ & $\kappa_{d}=2 / 7$ & Isentropic constant \\
\hline$F_{k}$ & $\begin{array}{l}\text { Thermal resistance due } \\
\text { to conduction }\end{array}$ & $\rho_{a}, \rho_{d}, \rho$ & $\begin{array}{l}\text { Moist and dry air density and } \\
\text { density of the air-spray mixture }\end{array}$ \\
\hline$k$ & Turbulent eddy viscosity & $\theta_{v}$ & Virtual temperature \\
\hline $\begin{array}{l}k_{1}=5.09 \\
\quad k_{2}=19.36\end{array}$ & $\begin{array}{l}\text { Constants entering the } \\
\text { experimental expression describing } \\
\text { the dependence of water vapor } \\
\text { pressure on temperature }\end{array}$ & $\begin{array}{c}\alpha=0.3, \alpha_{e}=1, \alpha_{\varepsilon}=0.77 \\
C_{\varepsilon, 1}=1.44, C_{\varepsilon, 2}=1.92\end{array}$ & $\begin{array}{l}\text { Coefficients entering the } \\
E-\varepsilon \text { turbulence model }\end{array}$ \\
\hline$p$ & $\begin{array}{l}\text { Production rate of turbulent } \\
\text { kinetic energy }\end{array}$ & $\varepsilon$ & $\begin{array}{l}\text { Dissipation rate of turbulent } \\
\text { kinetic energy }\end{array}$ \\
\hline
\end{tabular}

$$
\begin{aligned}
e & =\frac{1}{\alpha}, \quad \varepsilon=\frac{1}{k_{p} z}, \quad q=q_{\mathrm{sat}}\left(T_{s}\right)=\frac{1}{1-\pi_{9}}, \\
\pi_{1} \frac{d s}{d z} & =-\pi_{2} q_{w} s, \quad T_{a}=P=1, \\
\pi_{1} \frac{d T_{w}}{d z} & =\pi_{6}\left(T_{w}-1\right)-\pi_{7} q_{w}, \\
\text { at } \quad z & =z_{q, \theta} / z_{w}, \quad \\
u & =\frac{1}{k_{p}} \ln \frac{z_{w}}{z_{0}},
\end{aligned}
$$

at the wave crest level $z=1$ and

$$
\frac{d \varepsilon}{d z}=-\frac{\varepsilon}{z_{\infty}}, \quad k \frac{d s}{d z}+\pi_{1} s=0, \quad \theta=\frac{\theta_{\infty}}{T_{s}}, \quad q=\frac{q_{\infty}}{q_{0}},
$$

at the upper boundary of the physical domain of interest $z=z_{\infty} / z_{w}$. The derivation and discussion of these conditions is given in Rastigejev and Suslov (2016). Here $k_{p} \approx 0.43$ is the von Kármán constant. The roughness length $z_{0}$ and the friction velocity $u_{\star}$ are defined (Fairall et al. 2003; Rastigejev et al. 2011; Rastigejev and Suslov 2014) as

$$
z_{0}=0.015 \frac{u_{\star}^{2}}{g}, \quad u_{\star}=\sqrt{\frac{\tau_{0}}{\rho_{d 0}}},
$$

where $\tau_{0}$ is a given constant turbulent shear stress and $\rho_{d 0}$ is the density of dry air at the reference conditions. The quantity $S_{0}$ is the reference value for the water vapor saturation $S=\tilde{p}_{v} / \tilde{p}_{v \text {,sat }}$, where $p_{v}$ is the water vapor pressure and $p_{v \text {,sat }}$ is its saturated value; $\delta_{z-1}$ is the vertical distribution function of spray production with a sharp maximum at the nondimensional wave crest level $z=1 ; q_{\mathrm{sat}}$ is the specific humidity at saturation; $z_{q, \theta}$ and $z_{0}, z_{w}$, and $z_{\infty} \gg z_{w}$ are heat and momentum roughness lengths, wave crest level, and the altitude corresponding to the upper edge of the computational domain; $T_{s}$ is the sea surface temperature; $q_{0}$ is the reference specific humidity; $\theta_{\infty}$ and $q_{\infty}$ are the potential temperature and specific humidity at the upper edge $z_{\infty}$ of the computational domain, respectively. Their values are given in appendix A. Other model parameters not defined explicitly in the text are listed in Table 1.

Equations (1) and (2) represent mass conservation of sea spray and water vapor, respectively. Equations (3) and (4) describe a nonequilibrium heat exchange between the air and evaporating spray with the diffusion of heat between droplets and surrounding air represented by terms containing the temperature difference $\left(T_{a}-T_{w}\right) s$. While other authors attempted to account for the air-spray sensible and latent heat fluxes by using various empirical assumptions [e.g., the introduction of ad hoc heat source terms in Bianco et al. (2011)], in the current model this is done directly without any approximation of the heat exchange process between the two phases. Equations (5) and (6) represent a mixture-type $E-\varepsilon$ (also known as the $k-\varepsilon$ model) turbulence production/dissipation model widely used for practical calculations of various disperse two-phase flows. Finally, Eqs. (7) and (8) describe the vertical momentum flux and the hydrostatic balance in the atmosphere. Prior to proceeding with the further discussion of this model, we note that predictions based on its mechanical (isothermal) part were discussed and compared with the available field data in our previous publication Rastigejev and Suslov (2014) and here we focus mostly on its thermodynamic extension.

The definitions and physical meaning of nondimensional parameters (independent nondimensional groups) $\pi_{i}, i=1, \ldots, 14$, and their typical values are given in Table 1 in Rastigejev and Suslov (2016). ${ }^{1}$ In writing the model equations (1)-(12) here we introduce several simplifications compared with the original model

\footnotetext{
${ }^{1}$ Note a typographical error in Rastigejev and Suslov (2016): the definition of $\pi_{3}$ should read $\pi_{3}=3 D_{0} z_{w} \sigma s_{0}\left(1-S_{0}\right) /$ $\left(\alpha_{v} u_{\star} r^{2} F_{k 0} q_{0}\right)=5 \times 10^{-4}$.
} 
suggested in Rastigejev and Suslov (2016). We neglect the variation of the nondimensional coefficients of water vapor diffusion $D$ and thermal conductivity of air $K$ and of the specific latent heat of evaporation $\mathfrak{l}$ with air temperature by setting them to $D=K=\mathfrak{l}=1$ given a relatively small range of temperature variation $\lesssim 10 \mathrm{~K}$ in the considered physical domain $\sim 1 \mathrm{~km}$. We also neglect airdroplet heat transfer due to the water vapor diffusion from a cold droplet to warm surrounding air as it is much smaller than that due to thermal conductivity of air. This is achieved by formally setting $\pi_{4}=0$ in the governing equations given in Rastigejev and Suslov (2016).

The dimensional latent $\tilde{q}_{l}$ and sensible heat fluxes in air $\tilde{q}_{s a}$ and spray $\tilde{q}_{s w}$ above the wave crest level are defined as

$$
\begin{aligned}
\tilde{q}_{l} & =-\alpha_{v} u_{\star} \rho_{d 0} \mathfrak{l}_{0} q_{0} q_{l}, \quad \tilde{q}_{s a}=-\alpha_{T} u_{\star} c_{p d} \rho_{d 0} T_{s} q_{s a}, \\
\tilde{q}_{s w} & =-\alpha_{s} c_{w} u_{\star} \rho_{w} s_{0} T_{s} q_{s w},
\end{aligned}
$$

where $\alpha_{v}, \alpha_{T}$, and $\alpha_{s}$ are reciprocals of Schmidt numbers associated with turbulent diffusivity of water vapor, heat and spray, respectively; $c_{p d}$ and $c_{w}$ are specific heat capacities of dry air at constant pressure and liquid water, respectively; $u_{\star}, \mathfrak{l}_{0}$, and $\rho_{d 0}$ and $\rho_{w}$ are the friction velocity, specific heat of water evaporation at air temperature $T_{s}$ (used as the characteristic value of specific enthalpy of water evaporation), and densities of dry air at the sea surface level (which is used as characteristic value of air density) and liquid water, respectively. The nondimensional heat fluxes $q_{l}, q_{s a}$, and $q_{s w}$ appearing in Eq. (14) are defined as

$$
q_{l}=k \rho_{a} \frac{d q}{d z}, \quad q_{s a}=k \rho_{d} \frac{d \theta}{d z}, \quad q_{s w}=k s \frac{d T_{w}}{d z} .
$$

We also introduce the relative latent heat flux $\hat{q}_{l}$ and sensible heat fluxes $\hat{q}_{s a}$ and $\hat{q}_{s w}$ defined as the ratios of the corresponding heat fluxes to their absolute values in the spray-free reference atmosphere

$$
\begin{gathered}
\hat{q}_{l}=\frac{\tilde{q}_{l}\left(s_{0}\right)}{\left|\tilde{q}_{l}(0)\right|}=-\frac{\alpha_{v} q_{l}}{q_{\star}}, \quad \hat{q}_{s a}=\frac{\tilde{q}_{s a}\left(s_{0}\right)}{\left|\tilde{q}_{s a}(0)\right|}=-\frac{\alpha_{T} T_{s} q_{s a}}{\left|\theta_{\star}\right|}, \\
\hat{q}_{s w}=\frac{\tilde{q}_{s w}\left(s_{0}\right)}{\left|\tilde{q}_{s a}(0)\right|}=-\frac{\alpha_{s} c_{w} \rho_{w} s_{0} T_{s} q_{s w}}{c_{p d} \rho_{d 0}\left|\theta_{\star}\right|} .
\end{gathered}
$$

\section{b. Defining parameters}

In model (1)-(12) all $\pi$ coefficients depend on the values of the sea surface temperature $T_{s}$ and pressure $P_{s}$ as well as on the potential temperature $\theta_{\infty}$ and the specific humidity $q_{\infty}$ at the upper edge of a MABL, the friction velocity $u_{\star}$, and the wave height $z_{w}$. The definitions of nondimensional model parameters also involve sea spray characteristics: the average spray droplet radius $r$ and the rate of spray production that is proportional to the spray concentration $s_{0}$ at the wave crest level. Since there is a one-to-one correspondence between the values of $\theta_{\infty}$ and $\theta_{\star}$ and $q_{\infty}$ and $q_{\star}$ (see appendix A) for fixed values of $T_{s}$, $P_{s}$, and $u_{\star}$ and it is convenient to operate with $q_{\star}$ and $\theta_{\star}$ rather than with $q_{\infty}$ and $\theta_{\infty}$, we will use the former pair of parameters to describe the system. Therefore, the sprayladen atmosphere is described by parameters $\left(T_{s}, P_{s}, u_{\star}\right.$, $\left.\theta_{\star}, q_{\star}, s_{0}, r, z_{w}\right)$. Since it is often assumed that the spray production, the droplet size distribution, and significant wave height are determined by $u_{\star}$ (Pierson and Moskowitz 1964; Donelan et al. 1985; Monahan 1986; Wu 1993), the number of independent nondimensional parameters can be reduced by three to $\mathbf{p} \equiv\left(T_{s}, P_{s}, u_{\star}\right.$, $\left.\theta_{\star}, q_{\star}\right)$. This represents a minimal set of governing parameters that describe main features of MABL in hurricane conditions. Such a parametric reduction is a result of a dimensional analysis of the model equations representing fundamental physical conservation principles. It enables us to conduct a detailed sensitivity study using only modest computational resources. To do that we choose ranges of these parameters to be between some limiting values

$$
\begin{aligned}
& T_{s}^{l} \leq T_{s} \leq T_{s}^{u}, \quad P_{s}^{l} \leq P_{s} \leq P_{s}^{u}, \quad u_{\star}^{l} \leq u_{\star} \leq u_{\star}^{u}, \\
& \theta_{\star}^{l} \leq \theta_{\star} \leq \theta_{\star}^{u}, \quad q_{\star}^{l} \leq q_{\star} \leq q_{\star}^{u},
\end{aligned}
$$

so that they include the typical values for hurricanes:

$$
\begin{aligned}
& T_{s}^{l}=290 \mathrm{~K}, T_{s}^{u}=308 \mathrm{~K} ; \quad P_{s}^{l}=8 \times 10^{4} \mathrm{~Pa}, P_{s}^{u}=10^{5} \mathrm{~Pa} ; \\
& u_{\star}^{l}=1 \mathrm{~m} \mathrm{~s}^{-1}, u_{\star}^{u}=6 \mathrm{~m} \mathrm{~s}^{-1} ; \quad \theta_{\star}^{l}=-0.2 \mathrm{~K}, \theta_{\star}^{u}=0.2 \mathrm{~K}, \\
& q_{\star}^{l}=10^{-4}, q_{\star}^{u}=4 \times 10^{-4} .
\end{aligned}
$$

\section{Approximate self-similar solutions}

Model (1)-(12) offers an opportunity to further reduce a computational effort required for a systematic sensitivity study of spray parameterization via the use of the so-called approximate self-similarity of its solutions. This means that with a good accuracy the solution for the parameter set of interest $\mathbf{p}$ can be obtained by an algebraic rescaling of a known reference solution corresponding to some parametric set $\mathbf{p}_{0}$ without the need for obtaining a numerical solution of the complete problem for each individual set $\mathbf{p}$. This enables one to produce comprehensive physical results for a wide range of physical parameters at a fraction of a computational cost.

The main observation prompting such a powerful conclusion is that the latent and total sensible $\left(\tilde{q}_{s}=\tilde{q}_{s a}+\tilde{q}_{s w}\right)$ heat fluxes above the wave crest level can be approximated 
by stretching and shifting some universal function $\Phi$ (see appendix B for technical details)

$$
\begin{aligned}
& \tilde{q}_{l}(z ; \mathbf{p}) \approx c_{l}(\mathbf{p})+a(\mathbf{p}) \Phi[b(\mathbf{p}) z], \\
& \tilde{q}_{s}(z ; \mathbf{p}) \approx c_{s}(\mathbf{p})-a(\mathbf{p}) \Phi[b(\mathbf{p}) z],
\end{aligned}
$$

where $a(\mathbf{p}), b(\mathbf{p}), c_{l}(\mathbf{p})$, and $c_{s}(\mathbf{p})$ are scaling factors, and $z=\tilde{z} / z_{w}$. Therefore, the distributions of these heat fluxes can be scaled to the reference profiles obtained for some parametric values $\mathbf{p}=\mathbf{p}_{0}$ with accuracy $\varepsilon$ so that

$$
\begin{aligned}
& \max _{1 \leq \beta z \leq z_{\infty} / z_{w}}\left[\frac{\left|\tilde{q}_{l}\left(z ; \mathbf{p}_{0}\right)-\alpha \tilde{q}_{l}(\beta z ; \mathbf{p})-\gamma_{l}\right|}{\left|q_{l}\left(z ; \mathbf{p}_{0}\right)\right|}\right]<\varepsilon, \\
& \max _{1 \leq \beta z \leq z_{\infty} / z_{w}}\left[\frac{\left|\tilde{q}_{s}\left(z ; \mathbf{p}_{0}\right)-\alpha \tilde{q}_{s}(\beta z ; \mathbf{p})-\gamma_{s}\right|}{\left|q_{s}\left(z ; \mathbf{p}_{0}\right)\right|}\right]<\varepsilon,
\end{aligned}
$$

where

$$
\begin{aligned}
\alpha & =\frac{a\left(\mathbf{p}_{0}\right)}{a(\mathbf{p})}, \quad \beta=\frac{b\left(\mathbf{p}_{0}\right)}{b(\mathbf{p})}, \quad \gamma_{l}=c_{l}\left(\mathbf{p}_{0}\right)-\alpha c_{l}(\mathbf{p}), \\
\gamma_{s} & =c_{s}\left(\mathbf{p}_{0}\right)-\alpha c_{s}(\mathbf{p})
\end{aligned}
$$

and $z_{\infty} \sim 100 \mathrm{~m}$ for some ranges $R_{i}$ of parameters $p_{i}$

$$
\left|p_{i, 0}-p_{i}\right|<R_{i}, i=1, \ldots, \operatorname{dim}(\mathbf{p}) .
$$

It is convenient to choose the reference parametric set $\mathbf{p}_{0}$ so that $b\left(\mathbf{p}_{0}\right)$ defined in Eq. (B14) attains its minimum over the parametric ranges of interest and the scaling coefficient $\beta$ remains smaller than 1. Expressions (19)(22) then lead to practical representations

$\tilde{q}_{l}(z ; \mathbf{p}) \approx \frac{1}{\alpha} \tilde{q}_{l}\left(\frac{z}{\beta} ; \mathbf{p}_{0}\right)-\frac{\gamma_{l}}{\alpha}, \quad \tilde{q}_{s}(z ; \mathbf{p}) \approx \frac{1}{\alpha} \tilde{q}_{s}\left(\frac{z}{\beta} ; \mathbf{p}_{0}\right)-\frac{\gamma_{s}}{\alpha}$.

The accuracy of these expressions will be demonstrated in section 5 a.

\section{The reduced model}

The model described by Eqs. (1)-(8) is rather complicated algebraically. It generally requires a fully numerical treatment. Numerical solutions to the problem can be routinely obtained using standard ODE solvers available, for example, in MATLAB. However, such solutions, while accurate, can only be obtained for one parametric set at a time. Identifying the major physical trends using solutions obtained only for a discrete set of parameters can be not so obvious. Alternatively, approximate analytic expressions explicitly demonstrating the dependence of solutions on various parameters in selected limiting cases can be obtained using the so-called asymptotic expansions. For example, in Rastigejev and Suslov (2016) such an approach was used to determine the barometric variation of quantities characterizing the dry spray-free atmosphere. Here we will investigate the effects brought about by the evaporation of spray droplets. To do that we adopt a number of simplifying assumptions that we list and justify next.

We assume that the spray concentration is sufficiently small (see appendix C for quantitative estimations) so that we can disregard the effect of buoyancy of a sprayladen air on turbulence and the variation of the density of spray-laden layer in the lower part of a MABL $\tilde{z} \lesssim 100 \mathrm{~m}$. Such effects indeed are weak when the amount of spray injected into the atmosphere does not exceed $s_{0} \leqslant 10^{-4}$ (Rastigejev and Suslov 2014, 2016). Thus, we can take advantage of the known distributions of the turbulent energy $e$, its dissipation rate $\varepsilon$, and the eddy viscosity $k$ for a spray-free atmosphere (Rastigejev and Suslov 2014):

$$
e=\frac{1}{\alpha}, \quad \varepsilon=\frac{1}{k_{p} z}, \quad k=k_{p} z
$$

and focus solely on the thermodynamic influence of spray on heat fluxes. After adding Eq. (4) multiplied by $\pi_{5} / \pi_{6}$ and Eq. (3) and moving the source $\delta$ term into the left-hand side we obtain

$$
\begin{aligned}
& \frac{d}{d z}\left\{k \rho_{d} \frac{d \theta}{d z}+\frac{\pi_{5}}{\pi_{6}}\left[s k \frac{d T_{w}}{d z}+\pi_{1} H_{z-1}\left(T_{w}-1\right)\right]\right\} \\
& \quad=-\frac{\pi_{5} \pi_{7}}{\pi_{6}} q_{w} s,
\end{aligned}
$$

the solution of which can be written in the form

$$
\theta(z)=1+\frac{\pi_{5} \pi_{7}}{\pi_{6}} \theta_{1}(z), \quad T_{w}(z)=1+\frac{\pi_{5} \pi_{7}}{\pi_{6}} T_{w 1}(z)
$$

so that Eq. (25) becomes

$\frac{d}{d z}\left[k \rho_{d} \frac{d \theta_{1}}{d z}+\frac{\pi_{5}}{\pi_{6}}\left(s k \frac{d T_{w 1}}{d z}-\pi_{1} H_{z-1} T_{w 1}\right)\right]=-q_{w} s$.

Equation (27) shows that the second term in the lefthand side of Eq. (25) can be disregarded since it is small compared to the first one given that $\pi_{5} / \pi_{6}=\alpha_{s} c_{w} s_{0} \sigma /$ $\left(\alpha_{T} c_{p d}\right) \ll 1$ for $\sigma s_{0} \ll 1$, where $\alpha_{s} c_{w} /\left(\alpha_{T} c_{p d}\right) \sim 1$ and the water/air density ratio $\sigma \sim 10^{3}$. As a result the following reduced energy balance equation is obtained:

$$
\frac{d}{d z}\left(k \rho_{d} \frac{d \theta}{d z}\right)=-\frac{\pi_{5} \pi_{7}}{\pi_{6}} q_{w} s .
$$

The asymptotic solutions for the reduced model are given in appendix $\mathrm{C}$. 

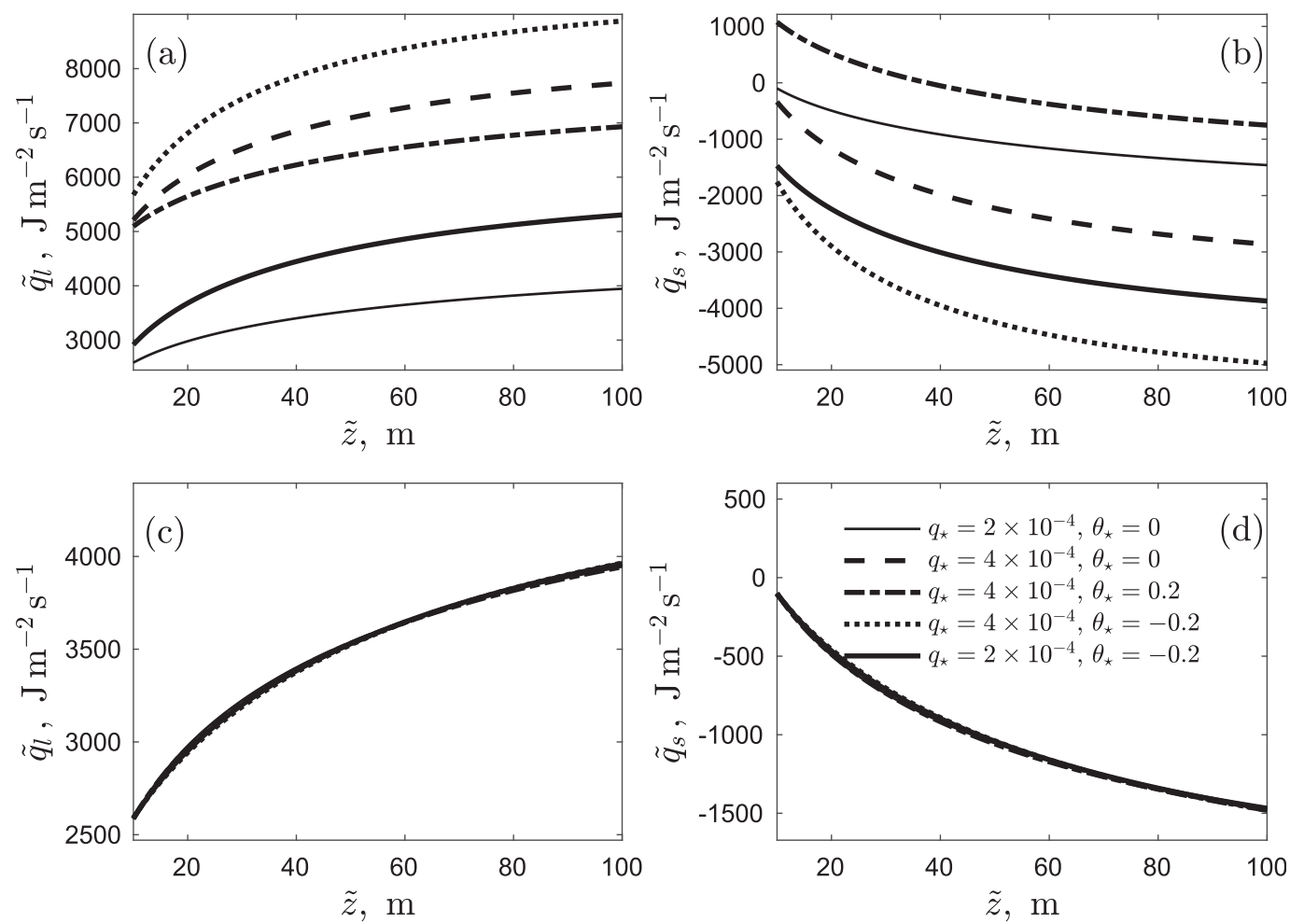

FIG. 1. Distributions of the (a),(c) latent $\tilde{q}_{l}$ and (b),(d) sensible $\tilde{q}_{s}$ heat fluxes for $s_{0}=1 \times 10^{-5}, r=200 \mu \mathrm{m}$, $u_{\star}=4 \mathrm{~m} \mathrm{~s}^{-1}$, and various values of $q_{\star}$ and $\theta_{\star}$. Panels (c) and (d) show the sensible and latent flux distributions, respectively, that are mapped onto the reference flux distributions corresponding to $q_{\star}=2 \times 10^{-4}$ and $\theta_{\star}=0 \mathrm{~K}$ (shown by the thin solid lines in the figure) using transformations (19) and (20).

\section{Results}

\section{a. Numerical results for approximate self-similar solutions}

We have confirmed approximate self-similarity of the vertical profiles of the latent and sensible heat fluxes in a series of numerical experiments for different values of $\mathbf{p}$ that are varied over their complete ranges [Eq. (18)]. Examples of these numerical experiments are presented in Figs. 1-3 for low and moderate spray concentrations $s_{0}=10^{-5}$ and $s_{0}=10^{-4}$, respectively. Figure 1 shows the vertical distributions of the latent and sensible heat fluxes for different values of parameters $q_{\star}$ and $\theta_{\star}$. Figure 2 demonstrates the same for different values of the parameters $T_{s}, P_{s}$, and $u_{\star}$. Similarly, Fig. 3 shows vertical profiles of the heat fluxes for different values of defining parameters p. For both values of spray concentrations the curves are mapped to the reference flux distributions for $T_{s}=300 \mathrm{~K}, P_{s}=10^{-5} \mathrm{~Pa}, u_{\star}=4 \mathrm{~m} \mathrm{~s}^{-1}$, $\theta_{\star}=0 \mathrm{~K}$, and $q_{\star}=2 \times 10^{-4}$ using transformations (19) and (20).

We have found that the accuracies of mapping are $\varepsilon \lessgtr 1 \%$ and $\varepsilon \lesssim 5 \%$ for $s_{0} \sim 10^{-5}$ and $s_{0} \sim 10^{-4}$, respectively. The discovered approximate self-similarity of heat flux profiles enables a significant speed-up of numerical estimations of heat fluxes via performing a simple mapping instead of solving the full problem for every set of defining parameters. This in turn can be used to construct an accurate and computationally efficient spray parameterization built into a larger NWP code.

\section{b. Reduced model}

To validate a numerical code, we have compared the analytical results for the reduced model and the corresponding numerical simulations using the full model. In particular, we examined the vertical profiles of the sensible and latent heat fluxes for both cases, see the left and right panels in Fig. 4, respectively. The lines (solid, dashed, and dotted) and markers (circles, squares, and triangles) correspond to the analytical and numerical solutions, respectively. The difference between the two sets of results was found not to exceed $1 \%$. Such a good agreement confirms high accuracy of the numerical code developed for computational simulation using the full model.

The asymptotic analysis shows that the spray and atmospheric boundary layer strongly affect each other's characteristics. It is known that the vertical 

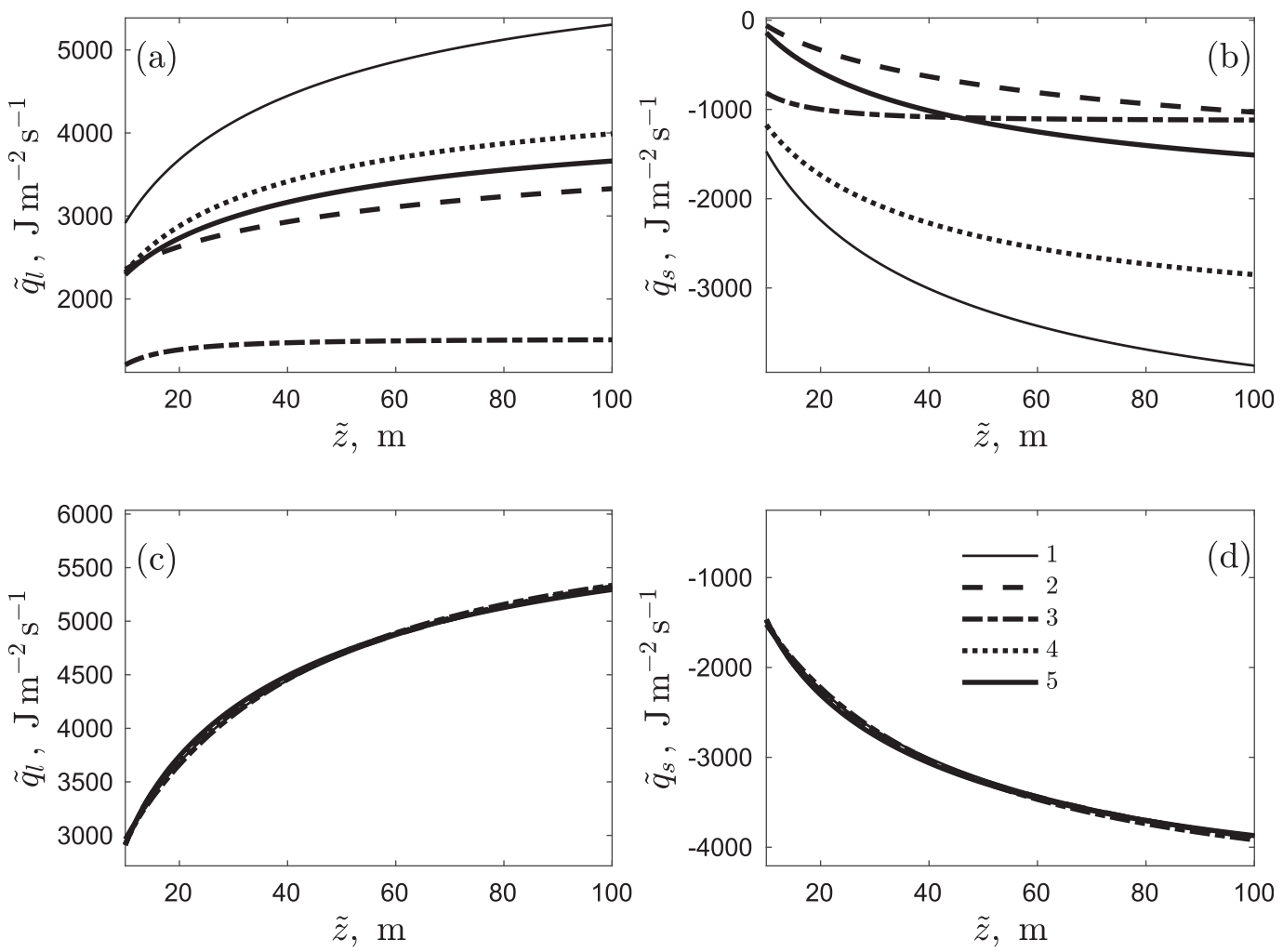

FIG. 2. Distributions of the (a),(c) latent $\tilde{q}_{l}$ and (b),(d) sensible heat fluxes $\tilde{q}_{s}$ for $s_{0}=10^{-5}, r=200 \mu \mathrm{m}$, and $q_{\star}=2 \times 10^{-4}$. The curves correspond to the following values of parameters: 1) $T_{s}=300 \mathrm{~K}, P_{s}=10^{5} \mathrm{~Pa}$, $u_{\star}=4 \mathrm{~m} \mathrm{~s}^{-1}, \theta_{\star}=-0.2 \mathrm{~K}$; 2) $T_{s}=308 \mathrm{~K}, P_{s}=10^{5} \mathrm{~Pa}, u_{\star}=4 \mathrm{~m} \mathrm{~s}^{-1}, \theta_{\star}=0 \mathrm{~K}$; 3) $T_{s}=308 \mathrm{~K}, P_{s}=8 \times 10^{4} \mathrm{~Pa}$, $u_{\star}=2 \mathrm{~m} \mathrm{~s}^{-1}, \theta_{\star}=-0.2 \mathrm{~K}$; 4) $T_{s}=308 \mathrm{~K}, P_{s}=8 \times 10^{4} \mathrm{~Pa}, u_{\star}=4 \mathrm{~m} \mathrm{~s}^{-1}, \theta_{\star}=-0.2 \mathrm{~K}$; and 5) $T_{s}=290 \mathrm{~K}$, $P_{s}=8 \times 10^{4} \mathrm{~Pa}, u_{\star}=4 \mathrm{~m} \mathrm{~s}^{-1}, \theta_{\star}=0 \mathrm{~K}$. Panels (c) and (d) show the sensible and latent flux distributions, respectively, that are mapped onto the reference flux (case 1 shown by the thin solid lines in the figure) using transformations (19) and (20).

distribution of the concentration of nonevaporating spray above the wave-crest level is given by $s(z)=z^{-\lambda}$ (Barenblatt and Golitsyn 1974; Rastigejev and Suslov 2014), while the air temperature $T_{a}(z)$ and the specific humidity $q(z)$ in a spray-free atmosphere vary logarithmically with the altitude. Here $\lambda=a /\left(k_{p} \alpha_{s} u_{\star}\right)$, where $a$ and $\alpha_{s}$ are the terminal speed of a droplet and the reciprocal of the Schmidt number based on the diffusivity of spray velocity, respectively. The asymptotic solutions in Eqs. (C7)-(C10) demonstrate that evaporating spray injected into a MABL modifies the vertical distributions $T_{a}(z)$ and $q(z)$ : additional terms containing $z^{-\lambda}$ appear in the corresponding expressions. In turn, the vertical moisture and air temperature profiles influence the distribution of the evaporating spray and cause the appearance of additional logarithmic terms, in particular, $C_{s 4} z^{1-\lambda} \ln z$. Note that this expression reduces to $\sim C_{s 4} \ln z$ for $\lambda \sim 1$. This term grows in magnitude with the rate of spray evaporation and can dominate the solution if evaporation is strong. This may explain the logarithmic profiles for spray concentration observed experimentally in OrtizSuslow et al. (2016).

\section{c. Spray influence on various atmospheric characteristics}

Figures 5 and 6 show the vertical distributions of quantities that influence heat transport in the sprayladen MABL for positive $\left(\theta_{\star}=0.2 \mathrm{~K}\right)$ and negative $\left(\theta_{\star}=-0.2 \mathrm{~K}\right)$ sensible heat fluxes, respectively, for droplet sizes ranging between 60 and $300 \mu \mathrm{m}$. Other parameters are fixed at $s_{0}=3 \times 10^{-5}, T_{s}=300 \mathrm{~K}$, $P_{s}=10^{5} \mathrm{~Pa}, u_{\star}=4 \mathrm{~m} \mathrm{~s}^{-1}$, and $q_{\star}=4 \times 10^{-4}$ in both cases. The data for the reference no-spray cases are shown by the thin solid lines.

As intuitively expected, the specific humidity $q$ decreases with the average droplet radius $r$ for the fixed value of the spray concentration $s_{0}$ since the combined surface area of droplets per unit volume of air defining the intensity of the droplet evaporation reduces for large droplets. The vertical profiles of the specific humidity are similar for both cases of the reference atmosphere, but 

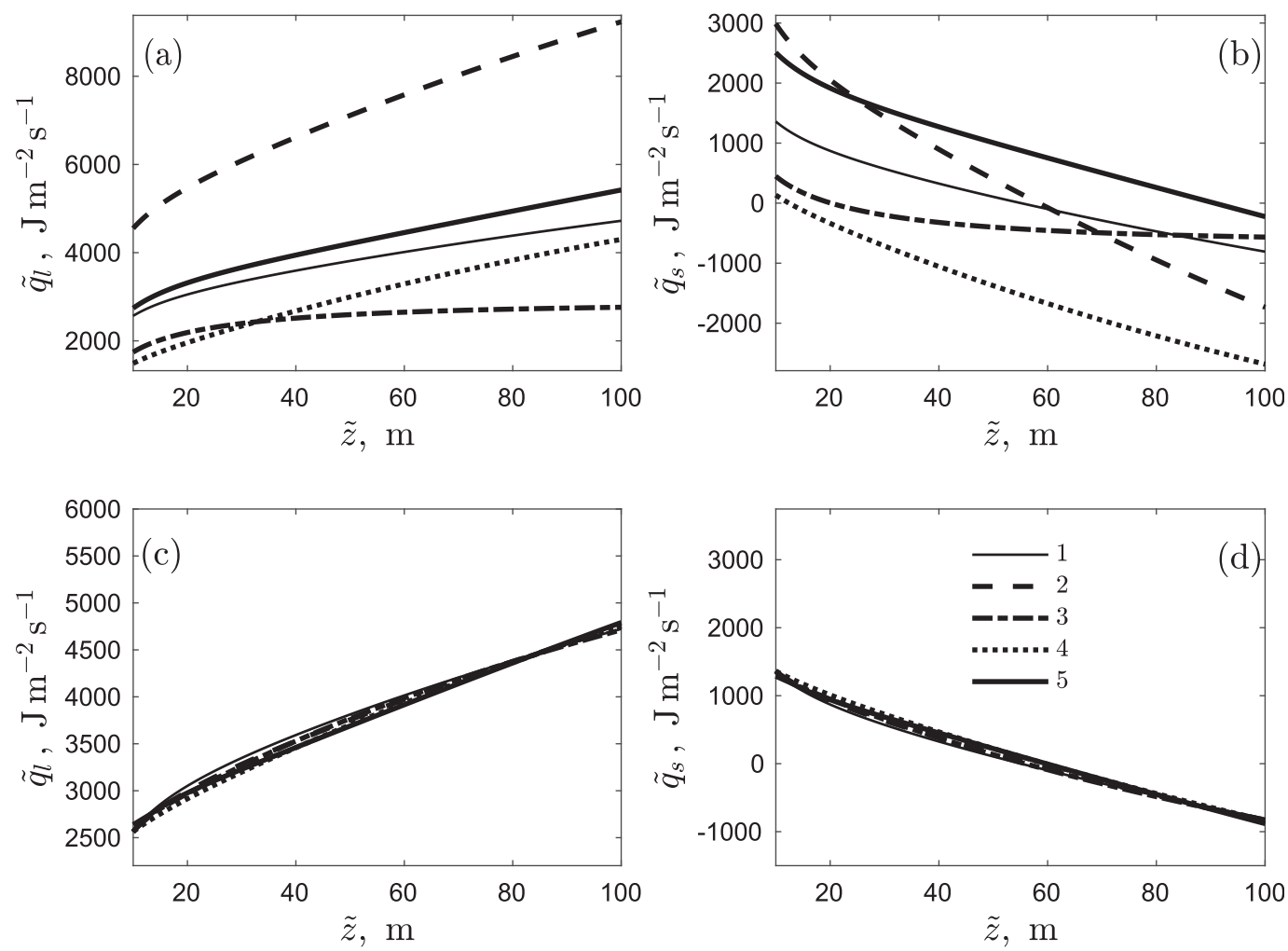

FIG. 3. Distributions of the (a),(c) latent heat fluxes $\tilde{q}_{l}$ and (b),(d) sensible heat fluxes $\tilde{q}_{s}$ for $s_{0}=10^{-4}$ and $r=$ $200 \mu \mathrm{m}$. Here curves correspond to the following values of parameters: 1) $T_{s}=300 \mathrm{~K}, P_{s}=10^{5} \mathrm{~Pa}, u_{\star}=4 \mathrm{~m} \mathrm{~s}^{-1}$, $\theta_{\star}=0 \mathrm{~K}, q_{\star}=2 \times 10^{-4}$; 2) $\left.T_{s}=308 \mathrm{~K}, P_{s}=10^{5} \mathrm{~Pa}, u_{\star}=4 \mathrm{~m} \mathrm{~s}^{-1}, \theta_{\star}=0 \mathrm{~K}, q_{\star}=4 \times 10^{-4} ; 3\right) T_{s}=290 \mathrm{~K}$, $P_{s}=8 \times 10^{4} \mathrm{~Pa}, u_{\star}=2 \mathrm{~m} \mathrm{~s}^{-1}, \theta_{\star}=0 \mathrm{~K}, q_{\star}=2 \times 10^{-4}$; 4) $T_{s}=308 \mathrm{~K}, P_{s}=8 \times 10^{4} \mathrm{~Pa}, u_{\star}=4 \mathrm{~m} \mathrm{~s}^{-1}, \theta_{\star}=-0.2 \mathrm{~K}$, $q_{\star}=2 \times 10^{-4}$; and 5) $T_{s}=290 \mathrm{~K}, P_{s}=8 \times 10^{4} \mathrm{~Pa}, u_{\star}=6 \mathrm{~m} \mathrm{~s}^{-1}, \theta_{\star}=0 \mathrm{~K}, q_{\star}=2 \times 10^{-4}$. Panels (c) and (d) show the sensible and latent flux distributions, respectively, that are mapped onto the reference flux distributions (case 1 shown by the thin solid lines in the figure) using transformations (19) and (20).

their values are somewhat higher for the reference atmosphere with the negative value of $\theta_{\star}$ due to the higher air temperature for a fixed sea surface temperature $T_{s}$ (and thus higher evaporation rate of droplets) in this case.

The vertical distributions of the air temperature $T_{a}$, the potential temperature $\theta$, and the virtual potential temperature $\theta_{v}$ are shown in Figs. 5a, 5d, 5g, and 6a, 6d, and $6 \mathrm{~g}$, respectively. The values of all temperatures decrease relative to their reference values in a spray-free atmosphere due to the evaporative cooling. The temperature differences between the spray-free and sprayaffected cases decrease with the droplet radius $r$ and are greater for the reference atmosphere with negative $\theta_{\star}$ reaching $\sim 10 \mathrm{~K}$ at the altitudes of $\sim 100 \mathrm{~m}$ for fine spray due to a more intensive evaporation of small droplets in a warm air. For spray containing medium-to-large droplets the distributions of all temperatures with the altitude $z$ generally follow the trends observed in the corresponding reference spray-free atmosphere: the temperatures decrease (increase) for the reference atmosphere with negative (positive) $\theta_{\star}$. However, these trends are altered by the presence of fine spray. For example, for $\theta_{\star}=-0.2 \mathrm{~K}$ and droplets with $r \lesssim 100 \mu \mathrm{m}$ the air temperatures decrease with the altitude in the lower atmosphere $z \lesssim 10$ (see Figs. 6b,d,g). Such a temperature decrease is caused by the air cooling due to intensively evaporating small droplets. Note that if $\theta_{\star}<0 \mathrm{~K}$ the air above the sea level is warmer than seawater when spray is present even though it is cooled by the spray at the sea level stronger relative to the reference spray-free atmosphere. The degree of thermodynamic nonequilibrium characterized by the difference $T_{a}-T_{w}$ between the air and spray temperatures increases with the droplet size (see Figs. $5 \mathrm{~h}$ and $6 \mathrm{~h}$ ). This is because large droplets have a weaker cooling effect on the surrounding air since their heat exchange with the ambient is hindered by a small surface-to-volume ratio.

The rate of TKE generation in the spray-free atmosphere is somewhat higher for the reference atmosphere with $\theta_{\star}=-0.2 \mathrm{~K}$ than that for $\theta_{\star}=0.2 \mathrm{~K}$ (compare Figs. $5 \mathrm{c}$ and $6 \mathrm{c}$ ). At first glance this may appear counterintuitive since a positive air buoyancy in the atmosphere 

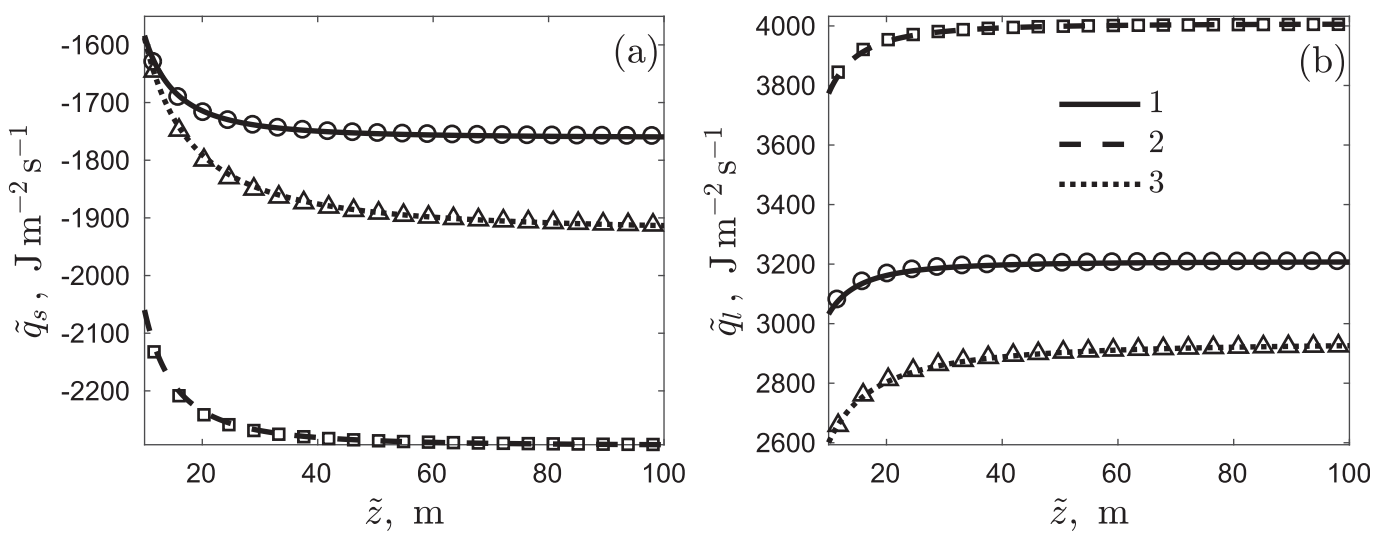

FIG. 4. Distributions of the (a) sensible $\tilde{q}_{s}$ and (b) latent $\tilde{q}_{l}$ heat fluxes for $s_{0}=3 \times 10^{-5}, u_{\star}=3 \mathrm{~m} \mathrm{~s}^{-1}, T_{s}=300 \mathrm{~K}$, and $P_{s}=10^{5} \mathrm{~Pa}$. The curves correspond to the following parameters: 1) $q_{\star}=2 \times 10^{-4}, \theta_{\star}=-0.1 \mathrm{~K}, r=500 \mu \mathrm{m}$; 2) $q_{\star}=2.5 \times 10^{-4}, \theta_{\star}=-0.15 \mathrm{~K}, r=500 \mu \mathrm{m}$; and 3) $q_{\star}=1.5 \times 10^{-4}, \theta_{\star}=-0.1 \mathrm{~K}, r=400 \mu \mathrm{m}$. The lines (solid, dashed, and dotted) and symbols (circles, squares, and triangles) depict asymptotic and numerical solutions, respectively.

with $\theta_{\star}=-0.2 \mathrm{~K}$ suppresses TKE, while a negative air buoyancy at $\theta=0.2 \mathrm{~K}$ encourages its production. However, these seemingly contradictory results are explained by the fact that the TKE production due to a vertical shear (which is $\sim \rho_{a}^{-2} \sim T_{a}^{2}$ ) is higher in the latter case. When a large amount of ocean spray is present, it strongly affects the vertical profile of TKE by modifying the buoyancy of the air-spray mixture in two different ways: thermodynamically by transforming the vertical distribution of the virtual potential temperature via the air-droplet heat and moisture exchange, and mechanically by changing the vertical airspray mixture density distribution (due to the decrease of the spray volume fraction in the vertical direction). As seen from Figs. 5b and 6b spray does not change the sign of the thermal stratification for any of the considered droplet sizes with the exception of small droplets at $\theta_{\star}=-0.2 \mathrm{~K}$. In this case fine spray does alter the stable thermal stratification existing in the reference atmosphere in the bottom $25-50 \mathrm{~m}$ of the atmospheric boundary layer, see the dashed and dashdotted lines in Fig. 6b.

It has been shown in Rastigejev and Suslov (2016) that the mechanical influence of spray on the TKE distribution is noticeably stronger than the thermodynamic one for droplets with $r \sim 100 \mu \mathrm{m}$ and the reference atmosphere with $\theta_{\star}=0 \mathrm{~K}$. Our present results shown in Figs. $5 \mathrm{c}$ and $6 \mathrm{c}$ demonstrate that this conclusion holds for the reference atmosphere in the complete range of parameter values typical for hurricanes. However, this finding does not fully corroborate with the assessment of the effect of large droplets on the vertical TKE distribution given in Bianco et al. (2011). There the authors concluded that large droplets increase turbulent mixing in the lower atmospheric layer by heating the air, which means that the thermodynamic influence of spray on the TKE distribution overpowers the mechanical one for such droplets. It is likely that this seemingly inaccurate conclusion was caused by the use of a turbulence model that does not fully account for the suppression of the turbulent energy by the ocean spray. Numerical computations show that the degree of TKE suppression due to the mechanical influence of spray strongly depends on the size of the droplets and the amount of spray: Figs. $5 \mathrm{c}$ and $6 \mathrm{c}$ demonstrate that the presence of even a small amount of fine spray has a significant turbulence suppression effect regardless of the values of $\theta_{\star}$ or $q_{\star}$.

The vertical distributions of spray volume fraction shown in Figs. 5e and 6e depend strongly on the droplet radius. This is because small droplets are transported by turbulent eddies to much higher altitudes than large ones and evaporate quicker due to a larger surface-tovolume ratio. The vertical profiles of spray temperature $T_{w}$ are presented in Figs. $5 \mathrm{f}$ and $6 \mathrm{f}$. The comparison of these plots shows that $T_{w}$ decreases slower with the altitude if $\theta_{\star}$ is small. In this case spray droplets are heated by a surrounding warm air at altitudes of $\sim 100 \mathrm{~m}$.

\section{d. Spray effect on vertical heat fluxes}

The vertical distributions of sensible heat fluxes in air $\tilde{q}_{s a}$ and spray $\tilde{q}_{s w}$ and latent $\tilde{q}_{l}$ and total $\tilde{Q}$ heat fluxes from the ocean to atmosphere are shown in Figs. 7 and 8 for the reference spray-free atmosphere with positive $\left(\theta_{\star}=0.2 \mathrm{~K}\right)$ and negative $\left(\theta_{\star}=-0.2 \mathrm{~K}\right)$ sensible heat fluxes, respectively, for the same values of parameters as in Figs. 5 and 6. As seen from Figs. 7a and 8a, the sensible heat flux in the liquid phase is greater for the atmosphere with a positive reference heat flux for droplets of the same sizes. This is due to the fact that the temperature $T_{w}$ of spray droplets decreases faster with the 
(a)

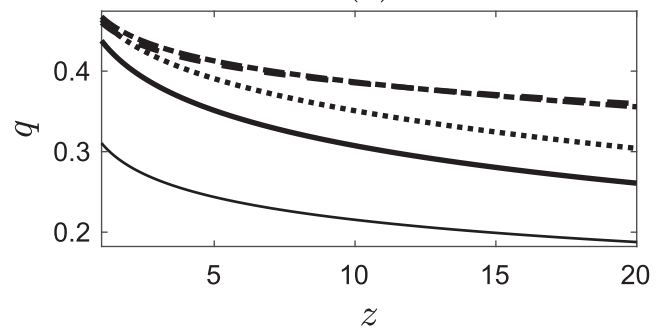

(c)

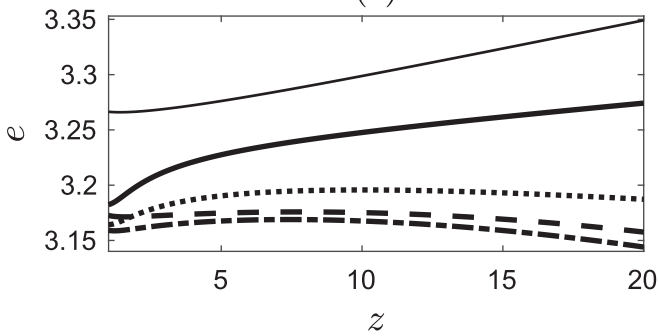

(e)

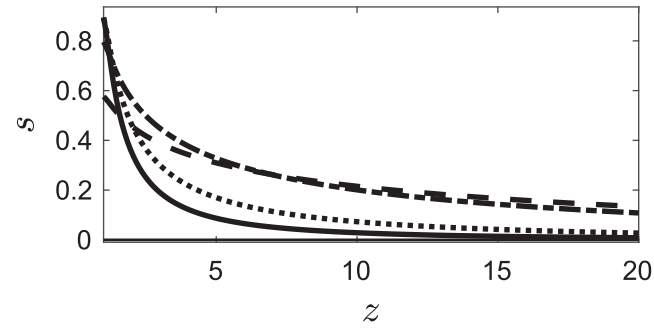

(g)

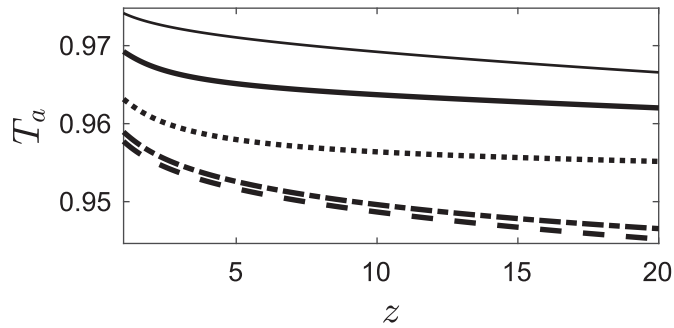

(b)

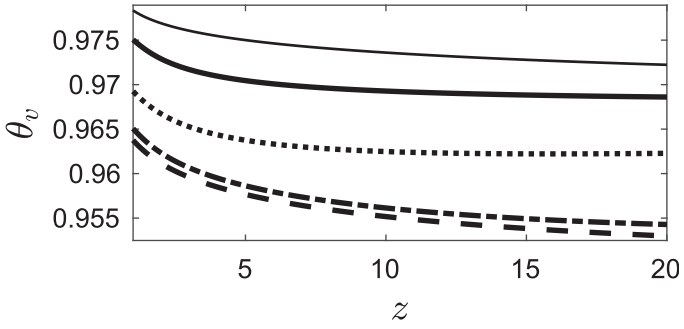

(d)

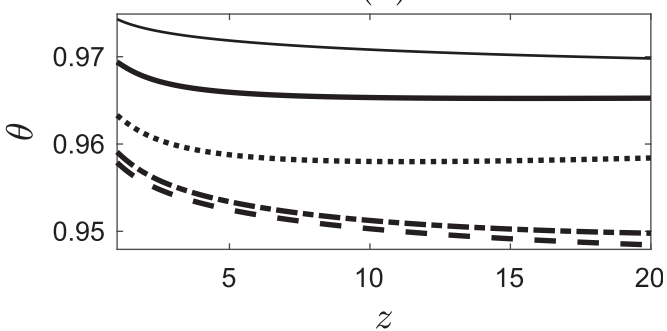

(f)

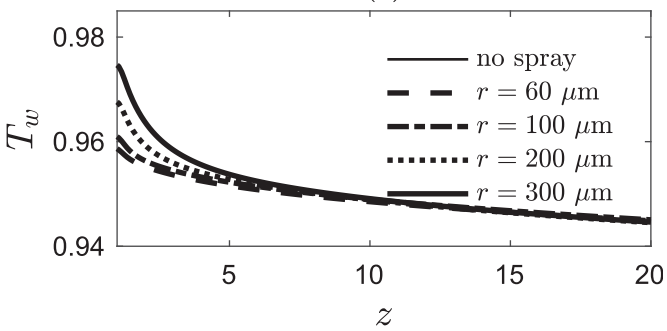

(h)

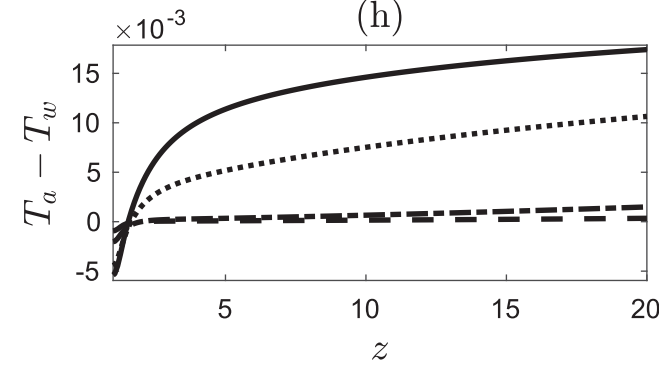

FIG. 5. Nondimensional (a) specific humidity $q$, (b) virtual potential temperature $\theta_{v}$, (c) turbulent kinetic energy $e$, (d) potential temperature $\theta$, (e) spray volume fraction $s$, (f) spray temperature $T_{w}$, (g) air temperature $T_{a}$, and (h) the difference $T_{a}-T_{w}$ between the air and spray temperatures computed for $T_{s}=300 \mathrm{~K}, P_{s}=10^{5} \mathrm{~Pa}$, $u_{\star}=4 \mathrm{~m} \mathrm{~s}^{-1}, \theta_{\star}=0.2 \mathrm{~K}, q_{\star}=4 \times 10^{-4}$, and various droplet sizes $r$.

altitude in this case (see Figs. $5 \mathrm{f}$ and $6 \mathrm{f}$ ). Note that the ratio of the sensible heat fluxes in the liquid phase

$$
\frac{\tilde{q}_{s w}\left(\tilde{z} ; \theta_{\star}>0, r\right)}{\tilde{q}_{s w}\left(\tilde{z} ; \theta_{\star}<0, r\right)}
$$

decreases with the droplet radius $r$ because heat exchange between warm air and cold spray droplets at high altitudes is less intensive for heavy droplets. As expected, $\tilde{q}_{s w}(\tilde{z})$ decreases slower with the vertical coordinate $\tilde{z}$ for small droplets since they are carried by turbulent eddies to higher altitudes. The overall observation is that the sensible heat flux $q_{s w}$ through the liquid phase comprises a nonnegligible fraction of the total heat flux especially near the ocean surface and omitting it from a model as often done may lead to a significant underprediction of the total heat flux values.

Droplets of all sizes noticeably redistribute the vertical heat flux in the air between its sensible and latent components. The vertical distribution of the air temperature $T_{a}$ is altered by spray so that the sensible heat flux in the air decreases with altitude for all values of 
(a)

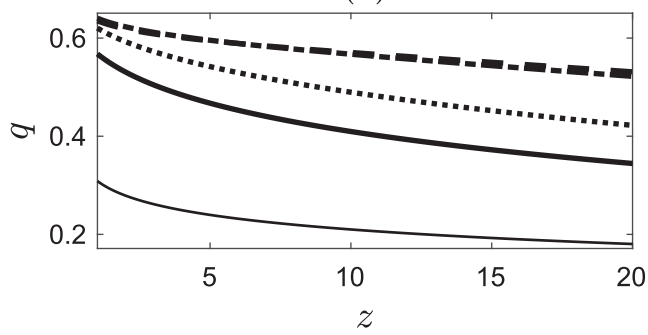

(c)

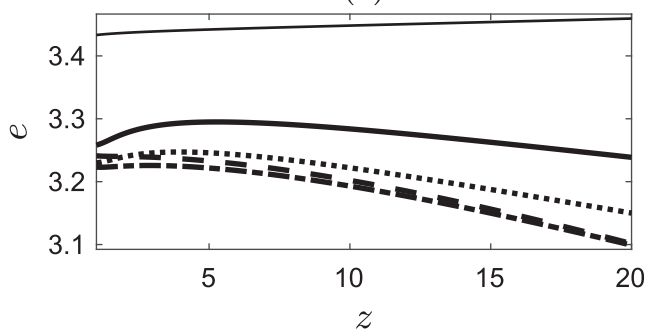

(e)

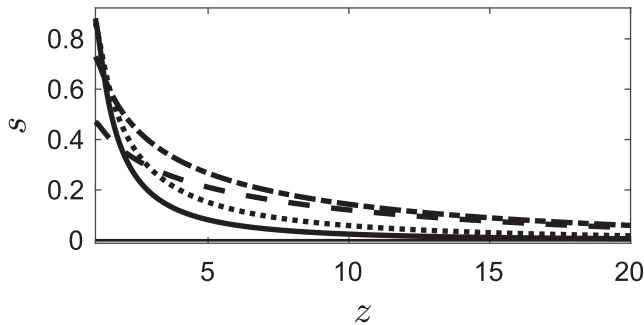

(g)

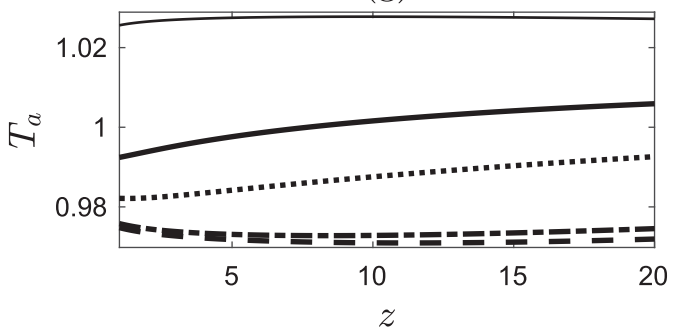

(b)

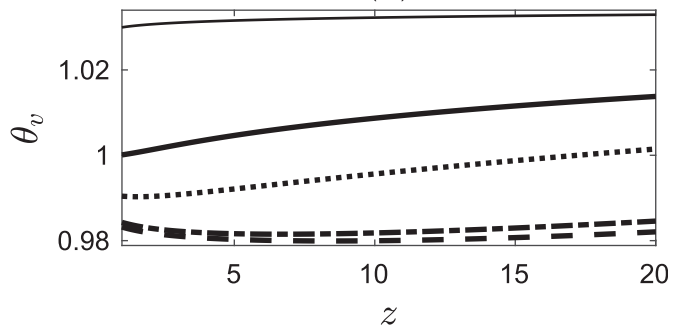

(d)

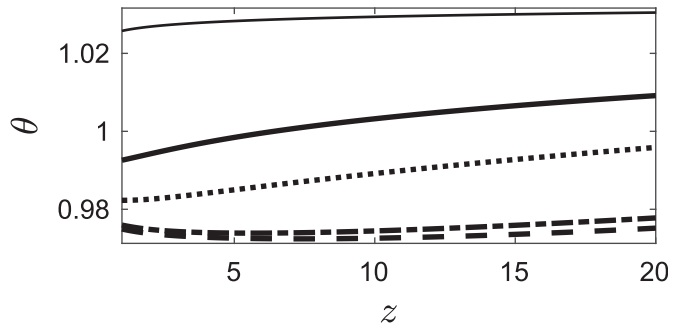

(f)

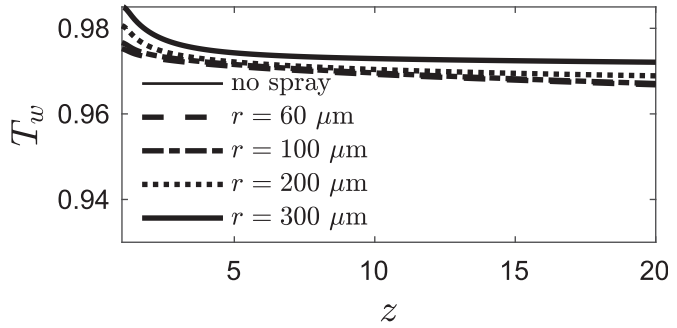

(h)

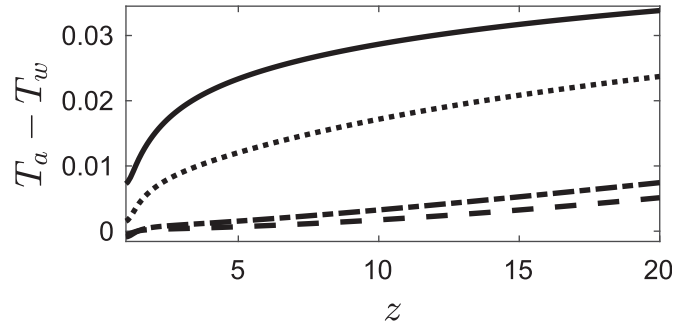

FIG. 6. As in Fig. 5, but for $\theta_{\star}=-0.2 \mathrm{~K}$.

parameters $\mathbf{p}$, spray concentration $s_{0}$, and droplet radius $r$. Since the total flux $\tilde{Q}(\tilde{z})$ remains constant above the wave crest level while the sensible heat $\tilde{q}_{s}(\tilde{z})$ decreases with the distance $\tilde{z}$ from the ocean surface, the latent heat flux $\tilde{q}_{l}(\tilde{z})=\tilde{Q}(\tilde{z})-\tilde{q}_{s}(\tilde{z})$ increases with $\tilde{z}$ (see Figs. 7c and $8 \mathrm{c}$ ).

The ocean spray has two opposing effects on the latent heat flux. On one hand, spray evaporates in the vicinity of the ocean surface effectively blocking the moisture transport from the ocean to the atmosphere and, as a result, reducing the latent heat flux. On the other hand, the evaporating spray increases the moisture gradient above wave crests and thus enhances the latent heat flux. The enhancing effect of spray dominates throughout the domain for large droplets with $r \geq 200 \mu \mathrm{m}$. It is also evident above a certain height that depends on $r$ (e.g., $40-60 \mathrm{~m}$ for $r=100 \mu \mathrm{m}$ ) for small droplets with $r<$ $200 \mu \mathrm{m}$ (see Figs. $7 \mathrm{c}$ and $8 \mathrm{c}$ ). This enhancing effect is stronger for heavy droplets (reaching its maximum strength for droplets with radii between 400 and $600 \mu \mathrm{m}$ ) because they are suspended and evaporate in a relatively thin layer above wave crests producing a large vertical moisture gradient (see Figs. 5a and 6a). Light droplets that are carried further away from the sea surface evaporate in a much thicker layer making the vertical moisture distribution more uniform and, thus, produce a weaker enhancing effect. Our finding that the vertical flux of water vapor near the ocean surface increases due 

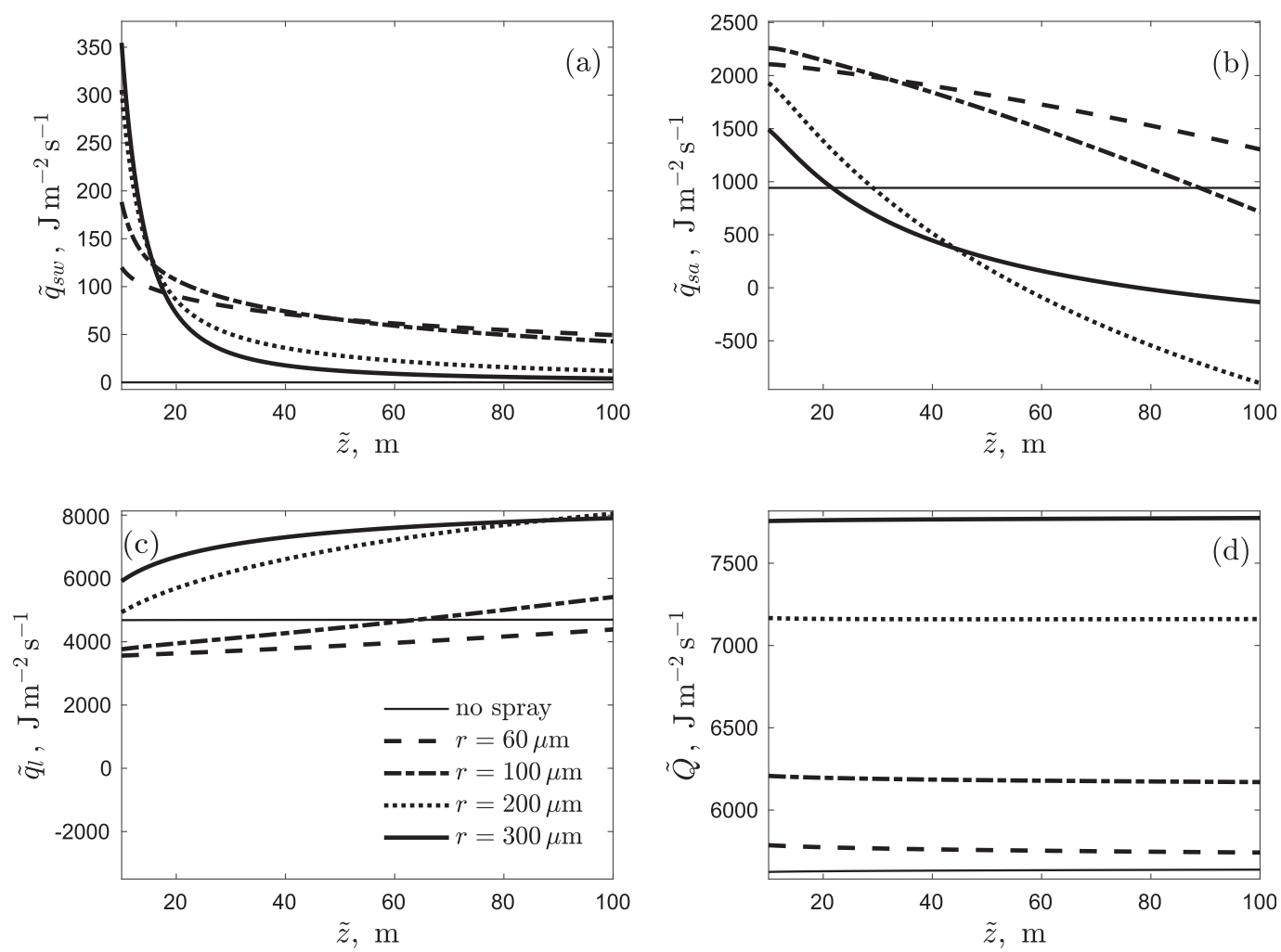

FIG. 7. Distributions of the sensible heat fluxes (a) $\tilde{q}_{s w}$ and (b) $\tilde{q}_{s a}$, (c) the latent heat flux $\tilde{q}_{l}$, and (d) the total heat flux $\tilde{Q}$ above wave crests for $s_{0}=3 \times 10^{-5}, T_{s}=300 \mathrm{~K}, P_{s}=10^{5} \mathrm{~Pa}, u_{\star}=4 \mathrm{~m} \mathrm{~s}^{-1}, q_{\star}=4 \times 10^{-4}, \theta_{\star}=0.2 \mathrm{~K}$, and various droplet sizes $r$.

to the spray presence only for large droplets does not fully support the assumption made in Andreas et al. (2015) that droplets of all sizes enhance this flux. This inaccurate premise is likely due to the fact that the empirical model presented in Andreas et al. (2015) does not consider the described above blocking effect of spray on the vertical water vapor transport.

The increase in water vapor production requires an additional supply of thermal energy that cannot be provided by the sensible heat flux from the water surface alone. Thus, the sensible heat flux in the air decreases with the vertical coordinate $\tilde{z}$ eventually becoming smaller than the reference one. Therefore, the energy required for droplet evaporation is received from the upper atmosphere. As seen from Figs. $7 d$ and $8 d$, the total heat flux $\tilde{Q}$ increases with the droplet radius $\tilde{r}$. This is the result of an intricate interplay between different spray influences on the MABL dynamics and structure.

Figures 9 and 10 show the vertical distributions of the relative sensible, latent and total heat fluxes defined by Eq. (16) for $\theta_{\star}=0.1 \mathrm{~K}$ and $\theta_{\star}=-0.1 \mathrm{~K}$, respectively. Numerical calculations show that spray redistributes heat flux between its sensible and latent components and enhances the total heat flux more efficiently for smaller values of the surface pressure and temperature. The total relative heat flux in the spray-laden MABL remains greater than 1 but its value is larger for an atmosphere with positive $\theta_{\star}$.

Figure 11 shows the total heat flux $\tilde{Q}$ versus the droplet radius $r$. The total heat flux also increases with the amount of spray suspended in the air (cf. the dashed and solid lines) and with the size of spray droplets. To understand why the total heat flux increases with droplet radius we need to consider the behavior of each of its components (the latent heat flux and the sensible heat fluxes in the gas and liquid phases) separately. As explained above, the latent heat flux increases in the presence of large droplets (see Figs. 7c and 8c) since the enhancing effect overpowers the blocking effect for such droplets. The thermal energy that leads to stronger water vapor production comes from the sea and the upper atmosphere. Transporting heat from the upper atmosphere is equivalent to the reduction of the vertical sensible heat flux in the air. Even though the reduction is stronger for large droplets (since they lead to a greater latent heat flux), it is also offset to a greater degree by the sensible heat transport from the ocean for such droplets. In other words, large droplets extract 

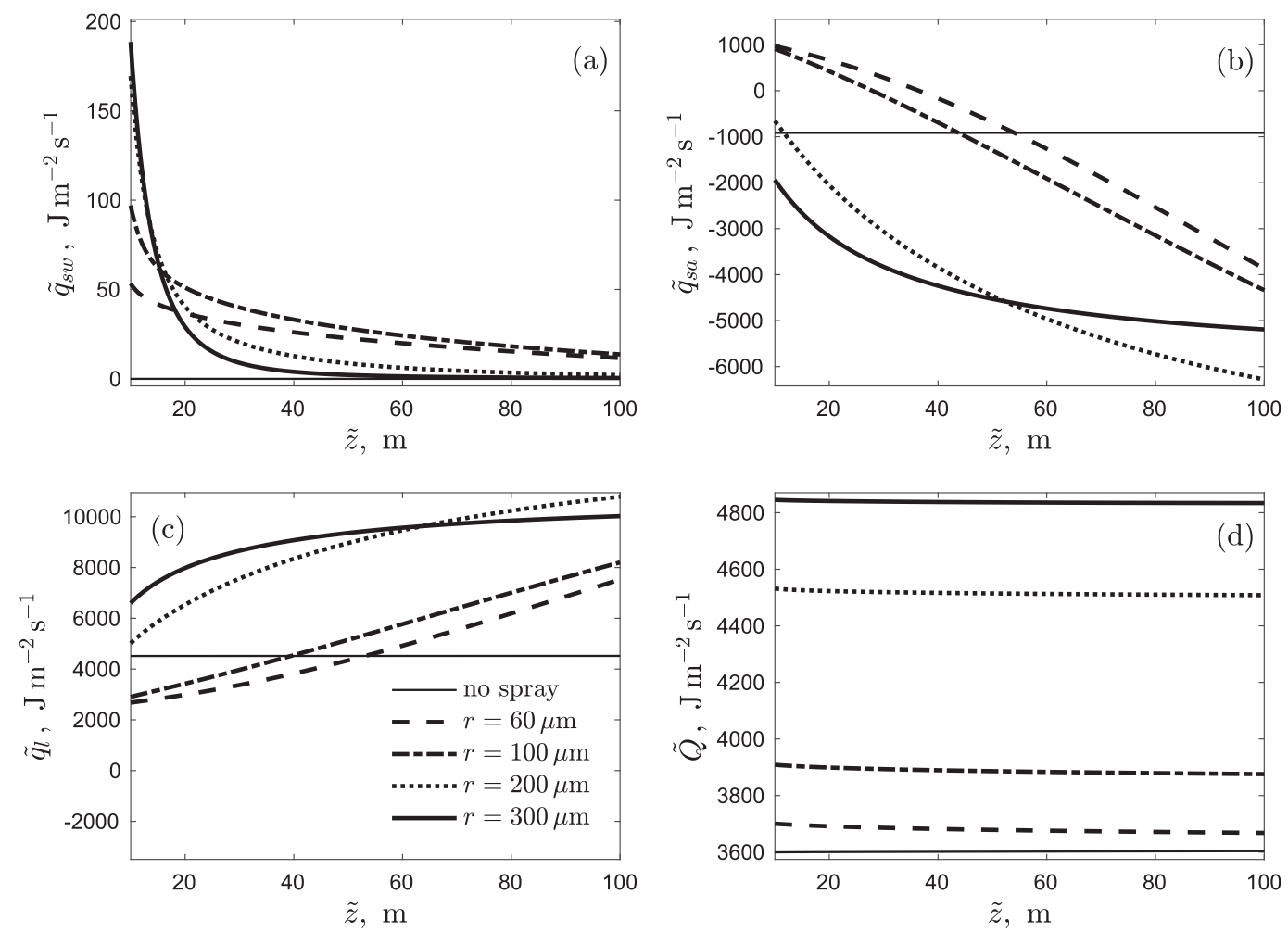

FIG. 8. As in Fig. 7, but for $\theta_{\star}=-0.2 \mathrm{~K}$.

sensible heat from the ocean more efficiently by creating a steeper air temperature gradient at the wave crest level. For example, the difference between the latent and sensible heat fluxes at the wave crest level for droplets with radii $r=200 \mu \mathrm{m}$ and $r=100 \mu \mathrm{m}$ are $\Delta \tilde{q}_{l} \equiv \tilde{q}_{l(r=200 \mu \mathrm{m})}-\tilde{q}_{l(r=100 \mu \mathrm{m})} \sim 1000 \mathrm{~J} \mathrm{~m}^{-2} \mathrm{~s}^{-1}$ and $\Delta \tilde{q}_{s a} \equiv \tilde{q}_{s a(r=200 \mu \mathrm{m})}-\tilde{q}_{s a(r=100 \mu \mathrm{m})} \sim-300 \mathrm{~J} \mathrm{~m}^{-2} \mathrm{~s}^{-1}$, respectively, for calculations presented in Fig. 7. This difference contributes $\Delta \tilde{q}_{l}+\Delta \tilde{q}_{s a} \sim 700 \mathrm{~J} \mathrm{~m}^{-2} \mathrm{~s}^{-1}$ toward the total heat flux for the larger droplets $(r=$ $200 \mu \mathrm{m}$ in this case). Another factor that contributes to the total heat flux increase with the droplet size, though to a lesser degree, is the sensible heat flux in the liquid phase that is greater for large droplets at the wave crest level due to somewhat larger concentration and larger spray temperature gradient in this area (see Figs. 7a and $8 \mathrm{a})$.

\section{e. Comparison with observations}

In this section we compare the dependence of the total vertical heat flux $\tilde{Q}$ on the 10 -m wind speed $\tilde{u}_{10}$ produced by the current model with the data presented in Bell et al. (2012) for wind speeds greater than $50 \mathrm{~m} \mathrm{~s}^{-1}$. It has been demonstrated in Bell et al. (2012) that the total vertical heat flux grows with the surface velocity as a power function $\sim \tilde{u}_{10}^{3.7}$. Further,
Richter and Stern (2014) noticed that this growth rate is much greater than a standard heat transfer scaling with wind speed $\sim \tilde{u}_{10}$ in a turbulent boundary layer. The authors suggested that this seemingly anomalous growth rate of the vertical heat flux with the wind speed may be due to the presence of ocean spray in MABL.

The influence of spray on the vertical heat flux depends strongly on its concentration (Rastigejev and Suslov 2016) and the average radius of its droplets (see Fig. 11). Unfortunately, neither of these two characteristics is known to an acceptable degree of certainty even for low wind speeds (Monahan 1986; Wu 1993; Andreas 1998; Anguelova et al. 1999; Fairall et al. 2009; Ortiz-Suslow et al. 2016). The spray production function for the high wind speeds $\tilde{u}_{10}>50 \mathrm{~m} \mathrm{~s}^{-1}$ that are of interest here is not known either. Nonetheless, the dependence of the spray production $f_{0}=a \rho_{w} s_{0}$ on the friction velocity $u_{\star}$ can be obtained from the wind energy balance considerations. It can be given by a power function

$$
f_{0}=A_{n} a \rho_{w} u_{\star}^{n},
$$

where the power coefficient $n$ varies between 3 and 5 depending on the mechanism of spray production 

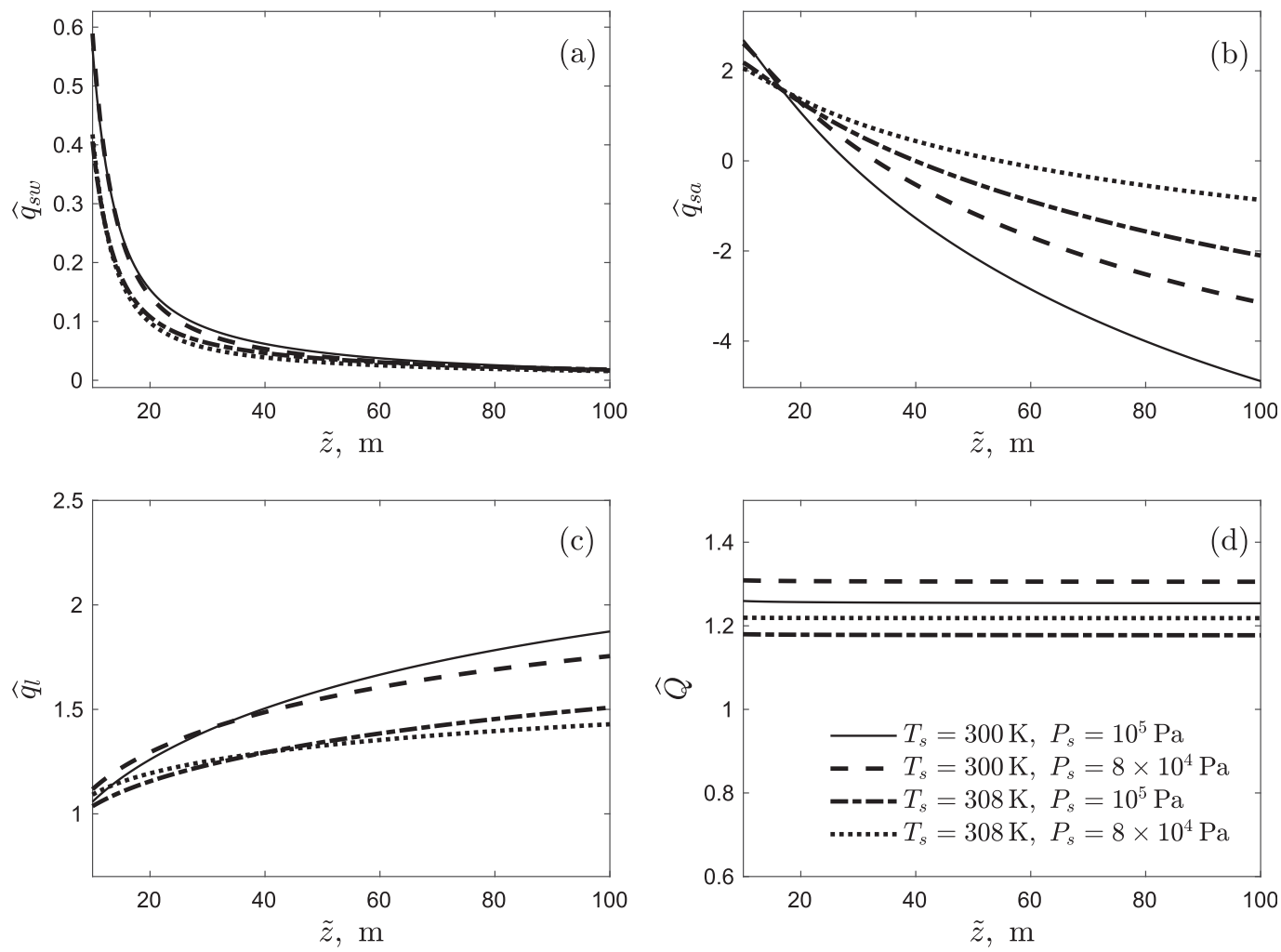

FIG. 9. Distributions of the relative sensible heat fluxes (a) $\hat{q}_{s w}$ and (b) $\hat{q}_{s a}$, (c) the relative latent heat flux $\hat{q}_{l}$, and (d) the relative total heat flux $\hat{Q}$ above wave crests for $s_{0}=3 \times 10^{-5}, u_{\star}=4 \mathrm{~m} \mathrm{~s}^{-1}, q_{\star}=4 \times 10^{-4}, \theta_{\star}=0.1 \mathrm{~K}$, $r=200 \mu \mathrm{m}$, and various values of the sea surface temperature $T_{s}$ and pressure $P_{s}$.

(Fairall et al. 2009). The average droplet radius is typically assumed to be $r=200 \mu \mathrm{m}$ (Fairall et al. 2009). Figure 12 shows the dependence of the total vertical heat flux on the friction velocity for the observational data (Bell et al. 2012) (squares) and that obtained with the present spray model for two cases: 1) $n=3$, $q_{\star}=5 \times 10^{-5}$ and 2) $n=5, q_{\star}=8.5 \times 10^{-5}$. The values of other parameters are the same for both cases: $\theta_{\star}=0, T_{s}=300 \mathrm{~K}$, and $P_{s}=10^{5} \mathrm{~Pa}$. The solid and dashed lines depict results for spray-laden MABLs. The values of parameter $A_{n}\left(A_{3}=3.9 \times 10^{-6} \mathrm{~s}^{3} \mathrm{~m}^{-3}\right.$ and $A_{5}=3.0 \times 10^{-8} \mathrm{~s}^{5} \mathrm{~m}^{-5}$ ) in Eq. (29) are chosen to match the numerically calculated heat fluxes with that derived from observations at the lowest wind velocity $\tilde{u}_{10}=52 \mathrm{~m} \mathrm{~s}^{-1}$. For comparison, heat fluxes in the reference spray-free boundary layer computed for the same values of $q_{\star}$ and identical boundary conditions are shown by the dash-dotted and dotted lines. The spray model results agree with the observational data for both spray production rates very well while heat fluxes are noticeably underestimated when spray is not present. This suggests that a fast growth of the vertical heat flux with the surface wind velocity is due to the strong influence of sea spray on MABL (Richter and Stern 2014).

\section{Conclusions}

In this work, we have studied the effect of sea spray on the vertical latent and sensible heat fluxes in a marine boundary layer for different droplet sizes, vertical distributions of air temperature, humidity, and turbulent intensity. We have performed numerical simulation and asymptotic analysis of the two-temperature nonequilibrium mathematical model of a marine boundary layer laden with evaporating spray taking into account the nonzero sensible heat flux in the reference spray-free atmosphere.

Consistent with previous studies of spray influence, notably of two-dimensional Lagrange-type numerical simulations reported, for example, in Shpund et al. (2014), the results of our analysis show that droplets of all sizes strongly affect the heat fluxes by redistributing the thermal energy between the latent and sensible components and enhancing the total and latent heat fluxes even when the spray concentration is relatively 

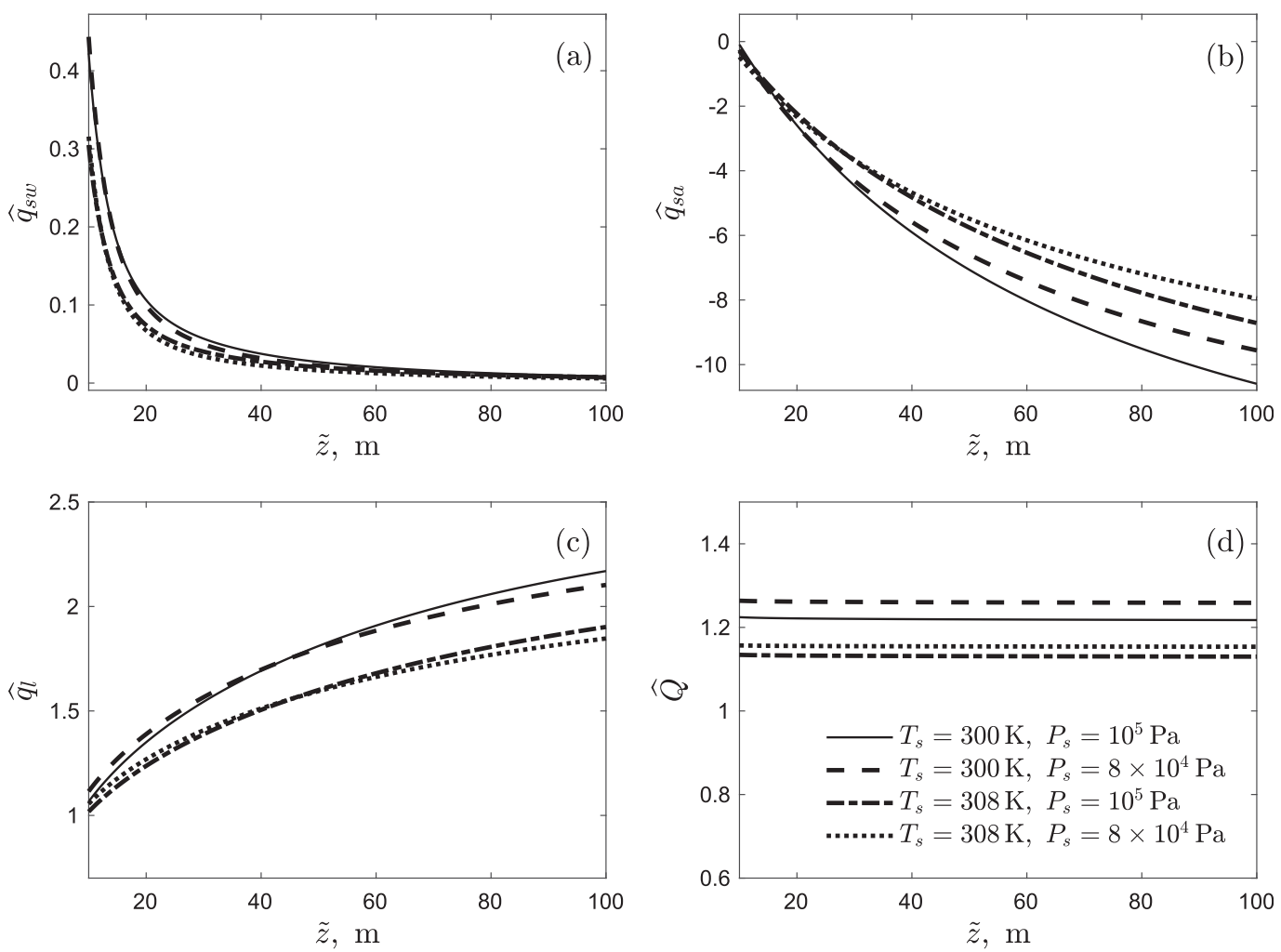

FIG. 10. As in Fig. 9, but for $\theta_{\star}=-0.1 \mathrm{~K}$.

low. The strength of the enhancing effect increases with the droplet radius reaching maximum at about $0.5 \mathrm{~mm}$. Qualitatively, this behavior does not depend on the vertical distribution of the air temperature or moisture. Furthermore, we have found that the spray affects the air buoyancy (thus the intensity of turbulent transport) primarily by causing nonuniform vertical density distribution rather than by modifying vertical profiles of the air temperature and humidity. Therefore, the coupling between thermodynamic and mechanical influences of
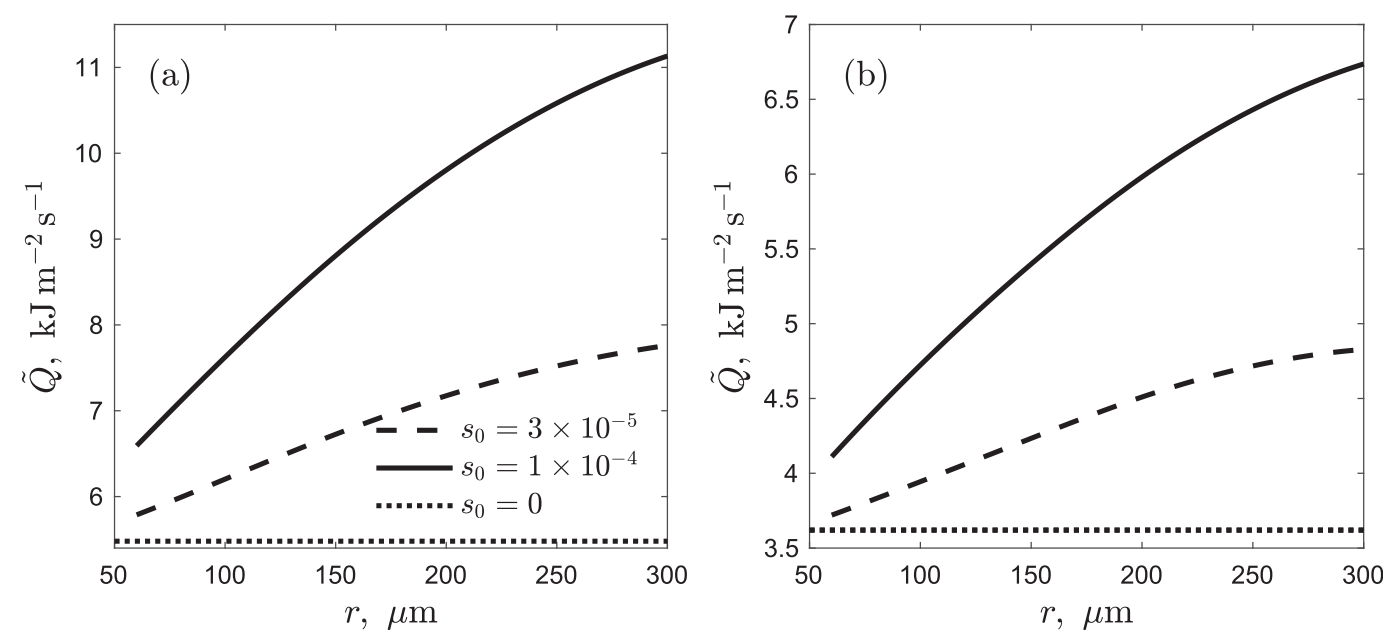

FIG. 11. Dependence of the total heat flux on the average droplet radius for (a) $\theta_{\star}=0.2 \mathrm{~K}$ and (b) $\theta_{\star}=-0.2$ $\mathrm{K}$. The values of parameters $T_{s}, P_{s}$, and $u_{\star}$ are the same as in Figs. 7 and 8 . The horizontal dotted lines correspond to the total heat flux values of $5480 \mathrm{~J} \mathrm{~m}^{-2} \mathrm{~s}^{-1}$ in (a) and $3620 \mathrm{~J} \mathrm{~m}^{-2} \mathrm{~s}^{-1}$ in (b) in the reference spray-free atmosphere. 


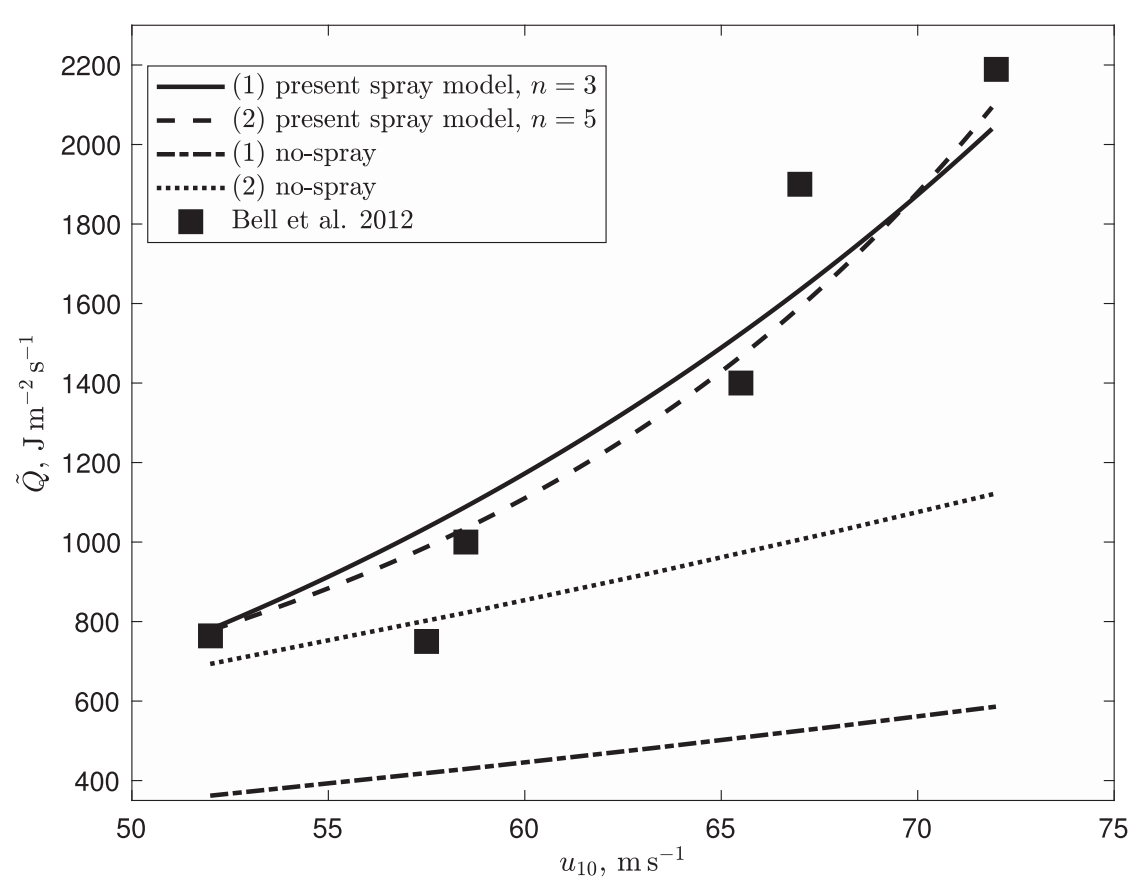

FIG. 12. Dependence of the total vertical heat flux on the surface wind velocity. The squares show results derived from the observational data (Bell et al. 2012). The lines correspond to results obtained using the present model for the spray-laden MABLs for 1) $n=3$, $q_{\star}=5 \times 10^{-5}$ and 2) $n=5, q_{\star}=8.5 \times 10^{-5}$ [see Eq. (29)] and the corresponding reference spray-free boundary layers.

spray is not symmetric: the spray-caused vertical density stratification reduces the vertical heat and momentum fluxes but the spray-caused thermal stratification does not noticeably affect these fluxes. The comparison of results produced using the present spray model with the available observation data indicates that the fast growth of the vertical heat flux with the surface wind speed could be explained by the presence of ocean spray.

We have also demonstrated that the vertical profiles of sensible and latent heat fluxes are approximately selfsimilar for a specified spray production intensity. This implies that the vertical heat profiles can be approximately calculated for a large range of vertical distributions of the air temperatures and humidity, and values of turbulent intensity by properly scaling a reference shape. The analytical solutions obtained via asymptotic analysis of the governing equations and/or scaling of the reference profiles will serve as a basis for accurate and efficient spray parameterization.

Acknowledgments. YR acknowledges support by a grant from the U.S. National Science Foundation under Award AGS-1832089 and support from ONR Summers 2017 and 2018 Faculty Research Programs.

\section{APPENDIX A}

\section{Calculation of $\boldsymbol{\theta}_{\infty}$ and $q_{\infty}$}

From equations for the nondimensional vertical fluxes of moisture and latent heat in the reference spray-free atmosphere, which are

$$
\frac{d \theta}{d z}=-\frac{\theta_{\star}}{\alpha_{T} k \rho_{d} T_{s}} \quad \text { and } \quad \frac{d q}{d z}=-\frac{q_{\star}}{\alpha_{\nu} k \rho_{a} q_{0}},
$$

we obtain the following expressions for $\theta_{\infty}$ and $q_{\infty}$

$$
\begin{aligned}
& \theta_{\infty}=T_{s}-\frac{\theta_{\star}}{\alpha_{T}} \int_{z_{q, \theta} / z_{w}}^{z_{\infty} / z_{w}} \frac{d z}{k \rho_{d}} \quad \text { and } \\
& q_{\infty}=q\left(T_{s}\right)-\frac{q_{\star}}{\alpha_{v}} \int_{z_{q, \theta} / z_{w}}^{z_{w}} \frac{d z}{k \rho_{a}} .
\end{aligned}
$$

The integrals and the values of $\theta_{\infty}$ and $q_{\infty}$ in Eq. (A2) are calculated by numerical integration of Eqs. (1)-(8) for the spray-free atmosphere $\left(s_{0}=0\right)$ with Eq. (A1) as the boundary conditions for $\theta$ and $q$ at some reference point $z=z_{\infty}$ far above the sea surface. Relationships (A2) show that there is a one-to-one correspondence between values $\theta_{\star}$ and $\theta_{\infty}$ and $q_{\star}$ and $q_{\infty}$ when other 
parameters are fixed. Therefore, either pair of parameters $\left(\theta_{\infty}, q_{\infty}\right)$ or $\left(\theta_{\star}, q_{\star}\right)$ can be utilized to describe the system dynamics. However, we found it more convenient to use the latter pair.

\section{APPENDIX B}

\section{Derivation of Approximate Self-Similar Vertical Distributions of Heat Fluxes}

Here we demonstrate that the vertical distributions of heat fluxes are approximately self-similar for $z_{w}<\tilde{z}<z_{\infty} \lesssim 100 \mathrm{~m}$. To do that we employ the reduced model described in section 4 that uses the water vapor conservation equation (2) in the form

$$
\frac{d}{d z}\left(z \frac{d q}{d z}\right)=\eta \frac{S-1}{1-S_{0}} s
$$

where

$$
\eta=\frac{\pi_{3}}{k_{p} \rho_{a}\left(F_{k}+F_{d}\right)}
$$

is a function of the air temperature $T_{a}$. Equation (B1) is a subject to the boundary conditions

$$
q\left(z_{q, \theta}\right)=q_{\mathrm{sat}}\left(T_{s}, P_{s}\right)=\frac{1}{1-\pi_{9}} \approx 1, \quad q\left(z_{\infty}\right)=\frac{q_{\infty}}{q_{0}} .
$$

Here we neglect the dependence of $\eta$, and $\rho_{a}$ on the air temperature $T_{a}$ by assuming that they are equal to some reference values that are taken at the wave crest level, $\eta=\eta\left(z_{w}\right)$ and $\rho_{a}=\rho_{a}\left(z_{w}\right)$. This assumption is justified by the observation that the values of $\eta$ and $\rho$ vary by relatively small amounts $(\sim 3 \%-4 \%)$ in the considered domain. Furthermore we use a simplified expression for the saturation ratio $S \approx q / q_{\text {sat }}$. This simplification introduces a relatively small error $\sim 2 \%$. For spray volume fraction $s$, the asymptotic expression (Rastigejev and Suslov 2014; Rastigejev et al. 2011) valid for small spray concentrations in Eq. (B1) is used

$$
s(z)=\left\{\begin{array}{cc}
z^{-\lambda}, & z>1, \\
1, & z \leq 1,
\end{array}\right.
$$

Since $\eta \ll 1$ for $s_{0} \lesssim 10^{-4}$, we take it as a small parameter and write the asymptotic solution of Eq. (B1) in the form

$$
q(z)=q^{0}(z)+\eta q^{1}(z)+\mathscr{O}\left(\eta^{2}\right) .
$$

The corresponding nondimensional latent heat flux is obtained by substituting Eq. (B5) into Eq. (15)

$$
q_{l}(z)=q_{l, 0}(z)+\eta q_{l, 1}(z)+\mathscr{O}\left(\eta^{2}\right) \approx q_{l, 0}(z)+\eta q_{l, 1}(z),
$$

where $q_{l, 0}$ and $q_{l, 1}$ are latent heat fluxes in the spray-free reference atmosphere and the main part of the additional latent flux due to spray is defined as

$$
q_{l, i}(z)=k_{p} z \frac{d q^{i}}{d z}, \quad i=1,2
$$

After substituting Eq. (B5) into Eq. (B1), applying boundary conditions in Eq. (B3), and equating the powers of $\eta$ we obtain the following problems:

$$
O(1): \frac{d}{d z}\left(z \frac{d q^{0}}{d z}\right)=0 ; \quad q^{0}\left(z_{q, \theta}\right)=1, \quad q^{0}\left(z_{\infty}\right)=q_{\infty} / q_{0} ;
$$

$O(\eta): \frac{d}{d z}\left(z \frac{d q^{1}}{d z}\right)=\frac{S^{0}-1}{1-S_{0}} s ; \quad q^{1}\left(z_{q, \theta}\right)=0, \quad q^{1}\left(z_{\infty}\right)=0$,

where $S^{0}=q^{0} / q_{\mathrm{sat}}^{0}$. The solution for the spray-free $O(1)$ problem reads

$$
q^{0}=1+c_{q 0} \ln \left(\frac{z z_{w}}{z_{q, \theta}}\right), \quad c_{q 0}=-\frac{q_{\star}}{\alpha_{v} k_{p} q_{0}}, \quad q_{l, 0}=-\frac{q_{\star}}{\alpha_{v} q_{0}} .
$$

The leading order expression

$$
S^{0}=1+C_{S} \ln \left(\frac{z z_{w}}{z_{q, \theta}}\right), \quad C_{S}=\left(k_{1}-k_{2}\right) c_{T 0}+c_{q 0},
$$

is obtained using the air temperature distribution $T_{a}^{0}=$ $1+c_{T 0} \ln \left(z z_{w} / z_{q, \theta}\right)$ in a spray-free atmosphere, where $c_{T 0}=-\theta_{\star} / \alpha_{T} k_{p} T_{0}$, the dependence of partial pressure of the saturated vapor on the air temperature in Eq. (10), and taking into account that $\left|c_{q 0}\right| \ll 1,\left|c_{T 0}\left(k_{1}-k_{2}\right)\right| \ll 1$, and $\pi_{8} \ll\left|c_{q 0}\right|$. After substituting Eqs. (B4) and (B11) into Eq. (B9) and integrating the equation we obtain the expression for the nondimensional latent heat flux above the wave crest level:

$$
q_{l, 1}(z)=q_{l, 1}(1)+\frac{k_{p} C_{S}}{1-S_{0}} \int_{1}^{z} \ln \left(\frac{z z_{w}}{z_{q, \theta}}\right) z^{-\lambda} d z .
$$

Next, we present expression (B12) in the form

$$
q_{l, 1}(z)=q_{l, 1}(1)+a_{1} \Phi(b z)-a_{1} \Phi(b),
$$

where 


$$
b=\frac{z_{w}}{z_{q, \theta}}, \quad a_{1}=\frac{k_{p} C_{S} b^{\lambda-1}}{1-S_{0}},
$$

and

$$
\Phi(b z)=\left\{\begin{array}{cc}
\frac{\ln (b z)(b z)^{1-\lambda}}{1-\lambda}-\frac{(b z)^{1-\lambda}}{(1-\lambda)^{2}}, & \lambda \neq 1, \\
\frac{1}{2} \ln ^{2}(b z), & \lambda=1 .
\end{array}\right.
$$

After substituting Eqs. (B10) and (B13) into Eq. (B6) and accounting for Eq. (14) we obtain Eq. (19), where $a=\alpha_{l} \eta a_{1}$ and $c_{l}=\alpha_{l}\left\{-q_{\star} /\left(\alpha_{v} q_{0}\right)+\eta\left[q_{l, 1}(1)-a_{1} \Phi(b)\right]\right\}$, $\alpha_{l}=-\alpha_{v} u_{\star} \rho_{d 0} \mathfrak{l}_{0} q_{0}$. It follows from Eqs. (14), (15), and (2)-(4) that

$$
\frac{d}{d z}\left(\tilde{q}_{l}+\tilde{q}_{s}\right)=0
$$

which implies Eqs. (19) and (20).

\section{APPENDIX C}

\section{Asymptotic Solutions}

We note that

$$
\pi_{3}=\lambda_{3} \pi_{2}, \quad \pi_{7}=\lambda_{7} \pi_{2},
$$

where

$$
\lambda_{3}=\frac{\sigma s_{0}}{q_{0}}, \quad \lambda_{7}=\frac{\mathfrak{l}_{0}}{c_{w} T_{s}} \approx 1.9 .
$$

Since $\pi_{2} \sim 10^{-3}-10^{-2}$ for typical spray droplet radii of the order of $100 \mu \mathrm{m}$ and saturation $S=0.5-0.9$ above the wave crest level, parameters $\pi_{3}$ and $\pi_{7}$ are also small provided $\lambda_{3} \lesssim 10$. This condition is satisfied if

$$
s_{0} \lesssim 10 \frac{q_{0}}{\sigma} \sim 2 \times 10^{-4},
$$

where the reference air humidity $q_{0} \sim 2 \times 10^{-2}$. Given that $\pi_{9} \sim q_{0}$, it can also be considered to be small. We also assume that barometric variations are small compared to variations caused by other physical effects. These observations enable us to develop an approximate asymptotic solution of Eqs. (1), (2), (8), and (25) by assuming that $\left(\pi_{2}, \pi_{3}, \pi_{5}, \pi_{7}, \pi_{8}, \pi_{9}\right)=\mathscr{O}(\varepsilon)$, where $\varepsilon$ is a formal small parameter, and by writing all quantities entering the equations in the form $f(z)=f_{0}(z)+\varepsilon f_{1}(z)+\mathscr{O}\left(\varepsilon^{2}\right)$. Substituting such formal expansions into the model equations we obtain a hierarchy of equations at different orders of $\varepsilon$ with the following leading order solutions below and above the wave crest level:

$$
s(z<1)=1+c_{s 1}+c_{s 2} z^{-\lambda}+c_{s 3} z+c_{s 4} z \ln z+\mathcal{O}\left(\varepsilon^{2}\right),
$$

$$
\begin{aligned}
q(z<1)= & \frac{1}{1-\pi_{9}}+c_{q 0} \ln \frac{z}{z_{\theta}}\left(1+\frac{1}{2} c_{T 0} \ln \frac{z}{z_{\theta}}\right)+c_{q 1} \\
& +c_{q 2} \ln z+c_{q 3} z+c_{q 4} \ln ^{2} z+c_{q 5} z \ln z+\mathscr{O}\left(\varepsilon^{2}\right),
\end{aligned}
$$

$$
\begin{aligned}
T_{a}(z<1)= & 1+c_{T 0} \ln \frac{z}{z_{\theta}}+c_{T 1}+c_{T 2} \ln z+c_{T 3} z \\
& +c_{T 4} z \ln z+\mathscr{O}\left(\varepsilon^{2}\right), \\
s(z \geq 1)= & C_{s 1}+C_{s 2} z^{-\lambda}+C_{s 3} z^{1-\lambda}+C_{s 4} z^{1-\lambda} \ln z \\
& +\mathscr{O}\left(\varepsilon^{2}\right),
\end{aligned}
$$

$$
\begin{aligned}
q(z \geq 1)= & \frac{1}{1-\pi_{9}}+c_{q 0} \ln \frac{z}{z_{\theta}}\left(1+\frac{1}{2} c_{T 0} \ln \frac{z}{z_{\theta}}\right)+C_{q 1} \\
& +C_{q 2} \ln z+C_{q 3} z+C_{q 4} \ln ^{2} z+C_{q 5} z^{1-\lambda} \ln z \\
& +C_{q 6} z^{1-\lambda}+\mathscr{O}\left(\varepsilon^{2}\right),
\end{aligned}
$$

$$
\begin{aligned}
T_{a}(z \geq 1)= & 1+c_{T 0} \ln \frac{z}{z_{\theta}}+C_{T 1}+C_{T 2} \ln z+C_{T 3} z \\
& +C_{T 4} z^{1-\lambda} \ln z+C_{T 5} z^{1-\lambda}+\mathcal{O}\left(\varepsilon^{2}\right), \text { and }
\end{aligned}
$$

$$
P(z)=1-\pi_{8}\left(z-z_{\theta}\right)+\mathscr{Q}\left(\varepsilon^{2}\right),
$$

where

$$
c_{T 0}=-\frac{\theta_{*}}{\alpha_{T} k_{p} T_{s}}, \quad c_{q 0}=-\frac{q_{*}}{\alpha_{v} k_{p} q_{0}} .
$$

The above approximate expressions are expected to be valid only in the lower atmosphere. The approximate validity range is determined as follows. Function (C7) describing the spray distribution above the wave crest level typically has a minimum at some large $z=z_{\min } \gg 1$. For $z>z_{\min }$ this function starts increasing, which is physically impossible. This is just an artifact of the low-order truncation of the approximate solution. Therefore, the above expressions can only be physically relevant for $z<z_{\min }$. Then the value of constant $C_{s 1}$ is set by requiring that $s\left(z_{\min }\right)=0$. Physically this means that we assume that see spray fully evaporates at $z=z_{\min }$ so that above this level the atmosphere remains spray-free. Subsequently, we require that solutions $(\mathrm{C} 4)-(\mathrm{C} 10)$ have values corresponding to the reference spray-free atmosphere at $z=z_{\min }$. Along with the boundary conditions at $z=z_{\theta}$ and matching (continuity of solutions) conditions at $z=1$ this defines all remaining constants in Eqs. (C4)-(C10). The expressions for constants appearing in the above solutions are too lengthy to be given 
here explicitly, but they are available on request in the form of a computer algebra (Wolfram Mathematica) code.

\section{REFERENCES}

Andreas, E. L, 1998: A new sea spray generation function for wind speeds up to $32 \mathrm{~m} \mathrm{~s}^{-1}$. J. Phys. Oceanogr., 28, 2175-2184, https:// doi.org/10.1175/1520-0485(1998)028<2175:ANSSGF > 2.0.CO;2.

_ and J. Decosmo, 1999: Sea spray production and influence on air-sea heat and moisture fluxes over the open ocean. Air-Sea Exchange: Physics, Chemistry and Dynamics, G. L. Geernaert, Ed., Springer, 327-362.

— and _ - 2002: The signature of sea spray in the hexos turbulent heat flux data. Bound.-Layer Meteor., 103, 303-333, https://doi.org/10.1023/A:1014564513650.

— , L. Mahrt, and D. Vickers, 2015: An improved bulk air-sea surface flux algorithm, including spray-mediated transfer. Quart. J. Roy. Meteor. Soc., 141, 642-654, https://doi.org/ 10.1002/qj.2424.

Anguelova, M., R. P. Barber, and J. Wu, 1999: Spume drops produced by the wind tearing of wave crests. J. Phys. Oceanogr., 29, 1156-1165, https://doi.org/10.1175/1520-0485(1999)029<1156: SDPBTW $>2.0 . \mathrm{CO} ; 2$.

Bao, J.-W., J. M. Wilczak, J.-K. Choi, and L. H. Kantha, 2000: Numerical simulations of air-sea interaction under high wind conditions using a coupled model: A study of hurricane development. Mon. Wea. Rev., 128, 2190-2210, https://doi.org/ 10.1175/1520-0493(2000)128<2190:NSOASI > 2.0.CO;2.

— C. W. Fairall, S. A. Michelson, and L. Bianco, 2011: Parameterizations of sea-spray impact on the air-sea momentum and heat fluxes. Mon. Wea. Rev., 139, 3781-3797, https://doi.org/ 10.1175/MWR-D-11-00007.1.

Barenblatt, G. I., and G. S. Golitsyn, 1974: Local structure of mature dust storms. J. Atmos. Sci., 31, 1917-1933, https://doi.org/ 10.1175/1520-0469(1974)031<1917:LSOMDS > 2.0.CO;2.

Bell, M. M., M. T. Montgomery, and K. A. Emanuel, 2012: Air-sea enthalpy and momentum exchange at major hurricane wind speeds observed during CBLAST. J. Atmos. Sci., 69, $3197-$ 3222, https://doi.org/10.1175/JAS-D-11-0276.1.

Bender, C. M., and S. A. Orszag, 1999: Advanced Mathematical Methods for Scientists and Engineers: Asymptotic Methods and Perturbation Theory. Springer, 593 pp.

Bianco, L., J.-W. Bao, C. W. Fairall, and S. A. Michelson, 2011: Impact of sea-spray on the atmospheric surface layer. Bound.-Layer Meteor., 140, 361-381, https://doi.org/10.1007/s10546-011-9617-1.

Bister, M., and K. A. Emanuel, 1998: Dissipative heating and hurricane intensity. Meteor. Atmos. Phys., 65, 233-240, https:// doi.org/10.1007/BF01030791.

Black, P. G., and Coauthors, 2007: Air-sea exchange in hurricanes. Synthesis of observations from the coupled boundary layer air-sea transfer experiment. Bull. Amer. Meteor. Soc., 88, $357-$ 374, https://doi.org/10.1175/BAMS-88-3-357.

Bortkovskii, R. S., 1987: Air-Sea Exchange of Heat and Moisture During Storms. E. C. Monahan, Ed., Springer, 194 pp.

Chen, Y., and X. Yu, 2016: Enhancement of wind stress evaluation method under storm conditions. Climate Dyn., 47, 3833-3843, https://doi.org/10.1007/s00382-016-3044-4.

Donelan, M. A., J. Hamilton, and W. H. Hui, 1985: Directional spectra of wind-generated ocean waves. Philos. Trans. Royal Soc. London, 315A, 509-562, https://doi.org/10.1098/rsta.1985.0054.

Drennan, W. M., J. A. Zhang, J. R. French, C. McCormick, and P. B. Black, 2007: Turbulent fluxes in the hurricane boundary layer. Part II: Latent heat fluxes. J. Atmos. Sci., 64, 1103-1115, https://doi.org/10.1175/JAS3889.1.

Edson, J. B., S. Anquetin, P. G. Mestayer, and J. F. Sini, 1996: Spray droplet modeling: 2. An interactive Eulerian-Lagrangian model of evaporating spray droplets. J. Geophys. Res., 101, 1279-1293, https://doi.org/10.1029/95JC03280.

Emanuel, K. A., 1986: An air-sea interaction theory for tropical cyclones. Part I: Steady-state maintenance. J. Atmos. Sci., 43, 585-604, https://doi.org/10.1175/1520-0469(1986)043<0585: AASITF $>2.0 . \mathrm{CO} ; 2$.

1995: Sensitivity of tropical cyclones to surface exchange coefficients and a revised steady-state model incorporating eye dynamics. J. Atmos. Sci., 52, 3969-3976, https://doi.org/ 10.1175/1520-0469(1995)052<3969:SOTCTS $>2.0$.CO;2.

1997: Some aspects of hurricane inner-core dynamics and energetics. J. Atmos. Sci., 54, 1014-1026, https://doi.org/ 10.1175/1520-0469(1997)054<1014:SAOHIC >2.0.CO;2.

Fairall, C. W., J. D. Kepert, and G. J. Holland, 1994: The effect of sea spray on surface energy transports over the ocean. Atmos. Ocean Syst., 2, 121-142.

, E. F. Bradley, J. F. Hare, A. A. Grachev, and J. B. Edson, 2003: Bulk parameterization of air-sea fluxes: Updates and verification for the COARE algorithm. J. Climate, 16, 571-591, https:// doi.org/10.1175/1520-0442(2003)016<0571:BPOASF >2.0.CO;2.

, M. L. Banner, W. L. Peirson, W. Asher, and R. P. Morison, 2009: Investigation of the physical scaling of sea spray spume droplet production. J. Geophys. Res., 114, C10001, https:// doi.org/10.1029/2008JC004918.

Gall, J. S., W. M. Frank, and Y. Kwon, 2008: Effects of sea spray on tropical cyclones simulated under idealized conditions. Mon. Wea. Rev., 136, 1686-1705, https://doi.org/10.1175/2007MWR2183.1.

He, H., Q. Wu, D. Chen, J. Sun, C. Liang, W. Lin, and Y. Xu, 2018: Effects of surface waves and sea spray on air-sea fluxes during the passage of typhoon Hagupit. Acta Oceanol. Sin., 37 (5), 1-7, https://doi.org/10.1007/s13131-018-1208-2.

Jakobsen, H., 2008: Chemical Reactor Modeling: Multiphase Reactive Flows. Springer, 1244 pp.

Jarosz, E., D. A. Mitchell, D. W. Wang, and W. J. Teague, 2007: Bottom-up determination of air-sea momentum exchange under a major tropical cyclone. Science, 315, 1707-1709, https://doi.org/10.1126/science.1136466.

Jeong, D., B. K. Haus, and M. A. Donelan, 2012: Enthalpy transfer across the air-water interface in high winds including spray. J. Atmos. Sci., 69, 2733-2748, https://doi.org/10.1175/JAS-D-11-0260.1.

Lighthill, J., 1999: Ocean spray and the thermodynamics of tropical cyclones. J. Eng. Math., 35, 11-42, https://doi.org/10.1023/A: 1004383430896.

Ling, S. C., and T. W. Kao, 1976: Parameterization of the moisture and heat transfer process over the ocean under whitecap sea states. J. Phys. Oceanogr., 6, 306-315, https://doi.org/10.1175/ 1520-0485(1976)006<0306:POTMAH >2.0.CO;2.

Magaritz, L., M. Pinsky, O. Krasnov, and A. Khain, 2009: Investigation of droplet size distributions and drizzle formation using a new trajectory ensemble model. Part II: Lucky parcels. J. Atmos. Sci., 66, 781-805, https://doi.org/10.1175/2008JAS2789.1.

Makin, V. K., 1998: Air-sea exchange of heat in the presence of wind waves and spray. J. Geophys. Res., 103, 1137-1152, https://doi.org/10.1029/97JC02908.

Monahan, E. C., 1986: The ocean as a source for atmospheric particles. The Role of Air-Sea Exchange in Geochemical Cycling, Springer, 129-163.

Mueller, J. A., and F. Veron, 2014: Impact of sea spray on air-sea fluxes. Part I: Results from stochastic simulations of sea spray 
drops over the ocean. J. Phys. Oceanogr., 44, 2817-2834, https://doi.org/10.1175/JPO-D-13-0245.1.

Ortiz-Suslow, D. G., B. K. Haus, S. Mehta, and N. J. M. Laxague, 2016: Sea spray generation in very high winds. J. Atmos. Sci., 73, 3975-3995, https://doi.org/10.1175/JAS-D-15-0249.1.

Paulsen, W., 2013: Asymptotic Analysis and Perturbation Theory. Chapman and Hall, 550 pp.

Peng, T., and D. Richter, 2017: Influence of evaporating droplets in the turbulent marine atmospheric boundary layer. Bound.-Layer Meteor., 165, 497-518, https://doi.org/10.1007/s10546-017-0285-7.

Perrie, W., W. Zhang, E. L. Andreas, W. Li, J. Gyakum, and R. McTaggart-Cowan, 2005: Sea spray impacts on intensifying midlatitude cyclones. J. Atmos. Sci., 62, 1867-1883, https:// doi.org/10.1175/JAS3436.1.

Pierson, W. J., Jr., and L. Moskowitz, 1964: A proposed spectral form for fully developed wind seas based on the similarity theory of S. A. Kitaigorodskii. J. Geophys. Res., 69, 5181-5190, https://doi.org/10.1029/JZ069i024p05181.

Pinsky, M., L. Mahgaritz, A. Khain, O. Krasnov, and A. Sterkin, 2008: Investigation of droplet size distributions and drizzle formation using a new trajectory ensemble model. Part I: Model description and first results in a nonmixing limit. J. Atmos. Sci., 65, 2064-2086, https://doi.org/10.1175/ 2007JAS2486.1.

Powell, M. D., P. J. Vickery, and T. A. Reinhold, 2003: Reduced drag coefficient for high wind speeds in tropical cyclones. Nature, 422, 279-283, https://doi.org/10.1038/nature01481.

Rastigejev, Y., and S. A. Suslov, 2014: model of spray-laden nearsea atmospheric layer in high wind conditions. J. Phys. Oceanogr., 44, 742-763., https://doi.org/10.1175/JPO-D-12-0195.1.

$\longrightarrow$, and —, 2016: Two-temperature non-equilibrium model of a marine boundary layer laden with evaporating ocean spray under high-wind conditions. J. Phys. Oceanogr., 46, 3083-3102, https://doi.org/10.1175/JPO-D-16-0039.1.

,-- , and Y.-L. Lin, 2011: Effect of ocean spray on vertical momentum transport under high-wind conditions. Bound.-Layer Meteor., 141, 1-20, https://doi.org/10.1007/s10546-011-9625-1.

Richter, D. H., and D. P. Stern, 2014: Evidence of spray-mediated air-sea enthalpy flux within tropical cyclones. Geophys. Res. Lett., 41, 2997-3003, https://doi.org/10.1002/2014GL059746.

Rouault, M. P., P. G. Mestayer, and R. Schiestel, 1991: A model of evaporating spray droplet dispersion. J. Geophys. Res., 96, 7181-7200, https://doi.org/10.1029/90JC02569.

Shpund, J., M. Pinsky, and A. Khain, 2011: Microphysical structure of the marine boundary layer under strong wind and spray formation as seen from simulations using a $2 \mathrm{D}$ explicit microphysical model. Part I: The impact of large eddies. J. Atmos. Sci., 68, 2366-2384, https://doi.org/10.1175/ 2011JAS3652.1.

—, J. A. Zhang, M. Pinsky, and A. Khain, 2012: Microphysical structure of the marine boundary layer under strong wind and spray formation as seen from simulations using a 2D explicit microphysical model. Part II: The role of sea spray. J. Atmos. Sci., 69, 3501-3514, https://doi.org/10.1175/JAS-D-11-0281.1.

,,--- , and -2014 : Microphysical structure of the marine boundary layer under strong wind and sea spray formation as seen from a 2D explicit microphysical model. Part III: Parameterization of height-dependent droplet size distribution. J. Atmos. Sci., 71, 1914-1934, https://doi.org/10.1175/ JAS-D-12-0201.1.

Stull, R. B., 1988: An Introduction to Boundary Layer Meteorology. Atmospheric and Oceanographic Sciences Library, Vol. 13, Springer, $670 \mathrm{pp}$.

Tang, S., Z. Yang, C. Liu, Y. H. Dong, and L. Shen, 2017: Numerical study on the generation and transport of spume droplets in wind over breaking waves. Atmosphere, $\mathbf{8}$, https:// doi.org/10.3390/atmos8120248.

Toffoli, A., A. V. Babanin, M. A. Donelan, B. K. Haus, and D. Jeong, 2011: Estimating sea spray concentration with the laser altimeter. J. Atmos. Oceanic Technol., 28, 1177-1183, https://doi.org/10.1175/2011JTECHO827.1.

Wang, Y., J. D. Kepert, and G. J. Holland, 2001: The effect of sea spray evaporation on tropical cyclone boundary layer structure and intensity. Mon. Wea. Rev., 129, 2481-2500, https://doi.org/ 10.1175/1520-0493(2001)129<2481:TEOSSE > 2.0.CO;2.

Wu, J., 1993: Production of spume drops by the wind tearing of wave crests: The search for quantification. J. Geophys. Res., 98, 18 221-18227, https://doi.org/10.1029/93JC01834.

Wu, L., A. Rutgersson, E. Sahlee, and X. G. Larsen, 2015: The impact of waves and sea spray on modelling storm track and development. Tellus, 67A, 27967, https://doi.org/10.3402/ tellusa.v67.27967.

Zhang, T., J. Song, S. Li, and L. Yang, 2016: The effects of winddriven waves and ocean spray on the drag coefficient and nearsurface wind profiles over the ocean. Acta Oceanol. Sin., 35 (11), 79-85, https://doi.org/10.1007/s13131-016-0950-6.

Zhang, W., and W. Perrie, 2008: The influence of air-sea roughness, sea spray, and storm translation speed on waves in North Atlantic storms. J. Phys. Oceanogr., 38, 817-839, https:// doi.org/10.1175/2007JPO3724.1. 